\title{
Standard Big-Bang Nucleosynthesis up to CNO with an improved extended nuclear network
}

\author{
Alain Coc \\ Centre de Spectrométrie Nucléaire et de Spectrométrie de Masse (CSNSM), CNRS/IN2P3, Université \\ Paris Sud, UMR 8609, Bâtiment 104, F-91405 Orsay Campus, France \\ Stéphane Goriely and Yi Xu \\ Institut d'Astronomie et d'Astrophysique, Université Libre de Bruxelles, CP 226, Boulevard du Triomphe, \\ B-1050 Bruxelles, Belgium \\ Matthias Saimpert and Elisabeth Vangioni \\ Institut d'Astrophysique de Paris, UMR 7095 CNRS, Université Pierre et Marie Curie, 98 bis Boulevard \\ Arago, Paris 75014, France
}

\begin{abstract}
Primordial or Big Bang nucleosynthesis (BBN) is one of the three strong evidences for the BigBang model together with the expansion of the Universe and the Cosmic Microwave Background radiation. In this study, we improve the standard BBN calculations taking into account new nuclear physics analyses and we enlarge the nuclear network until Sodium. This is, in particular, important to evaluate the primitive value of CNO mass fraction that could affect Population III stellar evolution. For the first time we list the complete network of more than 400 reactions with references to the origin of the rates, including $\approx 270$ reaction rates calculated using the TALYS code. Together with the cosmological light elements, we calculate the primordial Beryllium, Boron, Carbon, Nitrogen and Oxygen nuclei. We performed a sensitivity study to identify the important reactions for $\mathrm{CNO},{ }^{9} \mathrm{Be}$ and Boron nucleosynthesis. We reevaluated those important reaction rates using experimental data and/or theoretical evaluations. The results are compared with precedent calculations: a primordial Beryllium abundance increase by a factor of 4 compared to its previous evaluation, but we note a stability for $\mathrm{B} / \mathrm{H}$ and for the $\mathrm{CNO} / \mathrm{H}$ abundance ratio that remains close to its previous value of $0.7 \times 10^{-15}$. On the other hand, the extension of the nuclear network has not changed the ${ }^{7} \mathrm{Li}$ value, so its abundance is still $3-4$ times greater than its observed spectroscopic value.
\end{abstract}

Subject headings: primordial nucleosynthesis, nuclear reactions, abundances, cosmological parameters, early universe

September 15, 2018

\section{Introduction}

There are presently three observational evidences for the Big-Bang Model: the universal expansion, the Cosmic Microwave Background $(\mathrm{CMB})$ radiation and Primordial or Big-Bang $\mathrm{Nu}-$ cleosynthesis (BBN). The third evidence for a hot Big-Bang comes from the primordial abundances of the "light elements": ${ }^{4} \mathrm{He}, \mathrm{D},{ }^{3} \mathrm{He}$ and ${ }^{7} \mathrm{Li}$. They are produced during the first $\approx 20 \mathrm{~min}$ of the Universe when it was dense and hot enough for nuclear reactions to take place.

The number of free parameters entering in 
Standard BBN has decreased with time. The number of light neutrino families is known from the measurement of the $Z^{0}$ width by LEP experiments at CERN: $N_{\nu}=2.9840 \pm 0.0082$ LEP collaborations 2006). The lifetime of the neutron entering in weak reaction rate calculations and many nuclear reaction rates have been measured in nuclear physics laboratories. The last parameter to have been independently determined is the baryonic density of the Universe which is now deduced from the observations of the anisotropies of the CMB radiation coming from the WMAP satellite. The number of baryons per photon which remains constant during the expansion, $\eta$, is directly related to $\Omega_{\mathrm{b}}$ by $\Omega_{\mathrm{b}} \cdot h^{2}=3.65 \times 10^{7} \eta$. The WMAP7 gives now : $\Omega_{\mathrm{b}} \cdot h^{2}=0.02249 \pm 0.00056$ and $\eta=6.16 \pm 0.15 \times 10^{-10}$ (Komatsu et al. 2011). In this context, primordial nucleosynthesis is a parameter free theory and is the earliest probe of the Universe. We note an overall agreement except for the ${ }^{7} \mathrm{Li}$. In the literature many studies have been devoted to this lithium problem (Angulo et al. 2005; Coc et al. 2007; Cyburt et al. 2008; Chakraborty et al. 2011, and references therein) .

Deuterium, a very fragile isotope, is destroyed after BBN. Its most primitive abundance is determined from the observation of clouds at high redshift, on the line of sight of distant quasars. Very few observations of these cosmological clouds are available (Pettini et al. 2008, and references therein) and the adopted primordial D abundance is given by the average value:

$\mathrm{D} / \mathrm{H}=\left(2.82_{-0.19}^{+0.20}\right) \times 10^{-5}$.

After BBN, ${ }^{4} \mathrm{He}$ is still produced by stars. Its primitive abundance is deduced from observations in HII (ionized hydrogen) regions of compact blue galaxies. Galaxies are thought to be formed by the agglomeration of such dwarf galaxies which are hence considered as more primitive. The primordial ${ }^{4} \mathrm{He}$ abundance $Y_{p}$ (mass fraction) is given by the extrapolation to zero metallicity but is affected by systematic uncertainties (Aver et al. 2010; Izotov \& Thuan 2010) such as plasma temperature or stellar absorption. These most recent determinations based on almost the same set of observations lead to:

$Y_{p}=0.2561 \pm 0.0108$.

Contrary to ${ }^{4} \mathrm{He},{ }^{3} \mathrm{He}$ is both produced and de- stroyed in stars so that the evolution of its abundance as a function is subject to large uncertainties and has only been observed in our Galaxy (Bania et al. 2002),

${ }^{3} \mathrm{He} / \mathrm{H}=1.1 \pm 0.2 \times 10^{-5}$.

Consequently, the baryometric status of ${ }^{3} \mathrm{He}$ is not firmly established (Vangioni-Flam et al. 2003).

Primordial lithium abundance is deduced from observations of low metallicity stars in the halo of our Galaxy where the lithium abundance is almost independent of metallicity, displaying a plateau, the so-called Spite plateau (Spite \& Spite 1982). This interpretation assumes that lithium has not been depleted at the surface of these stars, so that the presently observed abundance is supposed to be equal to the initial one. The small scatter of values around the Spite plateau is an indication that depletion may not have been very effective.

Astronomical observations of these metal poor halo stars (Ryan et al. 2000) have led to a relative primordial abundance of:

$$
\mathrm{Li} / \mathrm{H}=\left(1.23_{-0.16}^{+0.34}\right) \times 10^{-10} .
$$

A more recent analysis by Sbordone et al. (2010) gives:

$$
\mathrm{Li} / \mathrm{H}=(1.58 \pm 0.31) \times 10^{-10}
$$

More generally, Spite \& Spite (2010) have reviewed the last Li observations and their different astrophysical aspects. See also Frebel \& Norris (2011) for a wide review.

In 2006, high-resolution observations of Li absorption lines in some very old halo stars have also claimed evidence for a large primitive abundance of the weakly-bound isotope ${ }^{6} \mathrm{Li}$ (Asplund et al. 2006). The ${ }^{6} \mathrm{Li} /{ }^{7} \mathrm{Li}$ ratios of $\sim 5 \times 10^{-2}$ were found to be about three orders of magnitude larger than the BBN-calculated value of ${ }^{6} \mathrm{Li} /{ }^{7} \mathrm{Li} \sim 10^{-5}$. The key $\mathrm{BBN}{ }^{6} \mathrm{Li}$ production mechanism is the $\mathrm{D}(\alpha, \gamma)^{6} \mathrm{Li}$ reaction at energies in the range of $50 \mathrm{keV} \leq E_{c m} \leq 400 \mathrm{keV}$ Serpico et al. 2004). This reaction has very recently been re-investigated (Hammache et al. 2010) confirming the previous result that Standard Big-Bang Nucleosynthesis cannot produce ${ }^{6} \mathrm{Li}$ at the required level. Concerning the ${ }^{6} \mathrm{Li}$ observational status, more recently, however, Cayrel et al. (2007) and Steffen et al. (2010) have pointed out that line asymmetries similar to those created by a ${ }^{6} \mathrm{Li}$ blend could also be produced by convective Doppler shifts in stellar atmospheres. In this con- 
text, these observations have to be confirmed. More detailed analyses are necessary to firmly conclude about the detection of the ${ }^{6} \mathrm{Li}$ abundance at this level in these metal poor stars (see Spite \& Spite 2010, for a review).

We consider in detail in this present study the other isotopes potentially produced by the Standard Big-Bang Nucleosynthesis including ${ }^{9} \mathrm{Be}$, ${ }^{10} \mathrm{~B},{ }^{11} \mathrm{~B}$ and the $\mathrm{CNO}$ isotopes. The production of ${ }^{9} \mathrm{Be},{ }^{10} \mathrm{~B}$ and ${ }^{11} \mathrm{~B}(\mathrm{BeB})$ and $\mathrm{CNO}$ isotopes have been studied in the context of standard and inhomogeneous BBN (Thomas et al. 1993, 1994; Kaijino et al. 1990; Kaijino \& Boyd 1990; Kajino 1995; Iocco et al. 2007, 2009). The most relevant analysis concerning $\mathrm{CNO}$ in $\mathrm{BBN}$ comes from Iocco et al. (2007) who included more than 100 nuclear reactions and predicted a $\mathrm{CNO} / \mathrm{H}$ abundance ratio of approximately $6 \times 10^{-16}$, with an upper limit of $10^{-10}$. These evaluations had been also performed to provide the initial conditions for the evolution of population III stars.

$\mathrm{BeB}$ nucleosynthesis is an important chapter of nuclear astrophysics. Specifically, rare and fragile nuclei, they are not generated in the normal course of stellar nucleosynthesis and are, in fact, destroyed in stellar interiors. This characteristic is reflected by the low abundance observations of these light species (Primas 2010; Boesgaard et al. 2010, and references therein). A glance to the abundance curve suffices to capture the essence of the problem: a gap separates He and C. At the bottom of this precipice rests the trio Li-Be-B. At very low metallicity, the $\mathrm{BeB}$ abundance is less than $10^{-12}$ relatively to hydrogen. Indeed, they are characterized by the simplicity of their nuclear structure (6 to 11 nucleons) and their scarcity in the Solar System and in stars. In fact, they are fragile because a selection principle at the nuclear level has operated in nature. Due to the fact that nuclei with mass 5 and 8 are unstable, BBN has almost stopped at $\mathrm{A}=7$, while nuclear burning in stars bypasses them through the triple-alpha reaction.

After BBN, the formation agents of $\mathrm{LiBeB}$ are Cosmic Rays interacting with interstellar or circumstellar CNO. Other possible origins have been also identified, for example supernova neutrino spallation (for ${ }^{7} \mathrm{Li}$ and ${ }^{11} \mathrm{~B}$ ). In contrast, ${ }^{6} \mathrm{Li},{ }^{9} \mathrm{Be}$ and ${ }^{10} \mathrm{~B}$ are pure cosmic-ray spallative products. (For a review see Vangioni-Flam et al. (2000).)
Recently, the LiBeB production has been considered in a cosmological context Rollinde et al. 2006, 2008). The non-thermal evolution with redshift of ${ }^{6} \mathrm{Li}, \mathrm{Be}$, and $\mathrm{B}$ in the first structures of the Universe has been studied. In this context cosmic rays are impinging alpha particles and $\mathrm{CNO}$ produced by the first massive stars, the so-called Population III stars. The computation has been performed in the framework of hierarchical structure formation and reliable ${ }^{6} \mathrm{Li}$ and $\mathrm{BeB}$ initial abundances coming from BBN are required to optimize the initial conditions.

Even though the direct detection of primordial CNO isotopes seems highly unlikely with the present observational techniques at high redshift, it is also important to better estimate their Standard Big-Bang Nucleosynthesis production. Hydrogen burning in the first generation of stars (Pop III stars) proceeds through the slow pp chains until enough carbon is produced (through the triplealpha reaction) to activate the $\mathrm{CNO}$ cycle. The minimum value of the initial $\mathrm{CNO}$ mass fraction that would affect Pop III stellar evolution is estimated to be $10^{-10}$ (Cassisi \& Castellani 1993) or even as low as $10^{-12}$ in mass fraction for the less massive ones (Ekström et al. 2008). This is only two orders of magnitude above the Standard BigBang Nucleosynthesis CNO yield using the current nuclear reaction rate evaluations of Iocco et al. (2007).

In addition, it has been shown that Pop III stars evolution is sensitive to the triple-alpha ${ }^{12} \mathrm{C}$ producing reaction and can be used to constrain the possible variation of the fundamental constants (Ekström et al. 2010). This reaction is sensitive to the position of the Hoyle state, which in turn is sensitive to the values of the fundamental constants. The amount of produced $\mathrm{CNO}\left({ }^{12} \mathrm{C}\right)$ could affect the $\mathrm{HR}$ diagram (CNO versus pp H-burning) and the final production of ${ }^{12} \mathrm{C}$ and ${ }^{16} \mathrm{O}$ in Pop III stars. Hence, it is important to quantify the amount of primordial CNO present at their birth. In the same context of the variations of the fundamental constants, ${ }^{8} \mathrm{Be}$ (which decays to two alpha particles within $\sim 10^{-16} \mathrm{~s}$ ) could become stable if these constants were only slightly different. At $\mathrm{BBN}$ time, this would possibly allow to bridge the "A=8 gap" and produce excess CNO. To determine how significant would be this excess, one needs to know the standard BBN production of 
the CNO elements.

Another motivation for this study is the above mentioned dichotomy concerning the Li abundance. At WMAP baryonic density, ${ }^{7} \mathrm{Li}$ is produced as ${ }^{7}$ Be that later decays. Nuclear ways to destroy this ${ }^{7}$ Be have been explored. An increased ${ }^{7} \operatorname{Be}(\mathrm{d}, \mathrm{p}) 2 \alpha$ cross section has been proposed by Coc et al. (2004) but was not confirmed by experiment described in Angulo et al. (2005) unless a new resonance is present (Cyburt \& Pospelov 2009) with very peculiar properties. Other ${ }^{7} \mathrm{Be}$ destruction channels have recently been proposed by Chakraborty et al. (2011) awaiting experimental investigation. Another scenario would be to take advantage of an increased late time neutron abundance. This is exactly what happens (in the context of varying constants) when the ${ }^{1} \mathrm{H}(\mathrm{n}, \gamma)^{2} \mathrm{H}$ rate is decreased. The neutron late time abundance is increased (with no effect on ${ }^{4} \mathrm{He}$ ) so that more ${ }^{7} \mathrm{Be}$ is destroyed by ${ }^{7} \operatorname{Be}(\mathrm{n}, \mathrm{p}){ }^{7} \operatorname{Li}(\mathrm{p}, \alpha) \alpha$. (see in Coc et al. 2007, Fig. 1).

The main difficulty in BBN calculations up to CNO is the extensive network needed, including n, p-, $\alpha-$, but also d-, t- and ${ }^{3} \mathrm{He}$-induced reactions. Most of the corresponding cross sections cannot be extracted from experimental data only. This is especially true for radioactive tritium-induced reactions, or for those involving radioactive targets like e.g. ${ }^{10}$ Be. For some reactions, experimental data, including spectroscopic data of the compound nuclei, are just inexistent. Hence, for many reactions, one has to rely on theory to estimate the reaction rates. Previous studies (Thomas et al. 1993, 1994; Iocco et al. 2007) have performed unpublished analyses of experimental data but have also apparently extensively used the prescription of Fowler \& Hoyle (1964) and Wagoner et al. (1967) to estimate many rates. These prescriptions often assume a constant astrophysical $S$-factor. This obviously cannot be a good approximation for most of the reactions considered in the BBN context. In this study we use at first more reliable rate estimates provided by the TALYS reaction code Goriely et al. 2008), next we perform a sensitivity study and, finally improve the rate estimates of the most important reactions by dedicated evaluations.

A detailed analysis of all reaction rates and associated uncertainties would be desirable but is unpractical for a network of $\approx 400$ reactions. So, in Section 2 we present the extended network and the standard thermonuclear reaction rates used. In Section 3, we study the sensitivity of the calculated primordial abundances up to $\mathrm{CNO}$ to variation of the rates by a factor of up to 1000 and reevaluate selected reaction rates. In Section 4, we present the BBN calculation results and we conclude in section 5 . Note that in the annex we give the full list of reactions with the references at the origin of the reaction rates.

\section{Nuclear cross section network}

In this study, we have included 59 nuclides from neutron to ${ }^{23} \mathrm{Na}$, linked by 391 reactions involving $\mathrm{n}, \mathrm{p}, \mathrm{d}, \mathrm{t}$ and ${ }^{3} \mathrm{He}$ induced reactions and $33 \beta$-decay processes. Reaction rates were taken primarily from Angulo et al. (1999); Descouvemont et al. (2004); Iliadis et al. (2010); $\mathrm{Xu}$ et al. (2010) and other evaluations when available. The complete list of reactions with associated references to the origin of the rates can be found in Table 4. Except for a few (historical) cases, the "direct reactions", listed in Table 4, are chosen to have positive Q-value. In our code, each of these reactions is systematically supplemented by the reverse reaction calculated according to the usual detailed balance prescription (Caughlan \& Fowler 1988; Angulo et al. 1999). In comparison with previous works, the present study includes two specific features, namely the introduction of the new evaluation of experimental reaction rates (NACRE 2) for target nuclei with $A<16$ and the extensive library of rates for experimentally unknown reactions, including all possible light particle captures. The latter library is based on the Hauser-Feshbach calculation with the TALYS reaction code Goriely et al. 2008) and though, a priori, the reaction model is not well suited for the description of the reaction mechanism on such light species, it appears to provide rather fair rates that can be used as a first guess for a sensitivity analysis, as detailed and discussed below.

\subsection{NACRE 2}

In the present paper, use is made of the updated NACRE 2 reaction rate evaluation. This new evaluation includes new rates for 15 charged-particle transfer reactions and for 19 capture reactions on 
stable targets with mass number $\mathrm{A}<16$. Compared to NACRE (Angulo et al. 1999), NACRE 2 features in particular (i) the addition to the collected NACRE experimental data of all the postNACRE ones published in refereed journals till 2010; (ii) the extrapolation of astrophysical $S$ factors to very low energies based on theoretical models, namely the distorted wave Born approximation (DWBA) for transfer reactions and the potential model for radiative captures (Xu et al. 2011). These models are simple albeit trustworthy for the considered reactions most likely dominated by a direct (rather than a compound) contribution. The experimental data available (usually well above the energy region of astrophysical interest) are fitted by spline interpolations. If narrow resonances happen to contribute, they are approximated by the single-level Breit-Wigner formula with varying particle widths. The HauserFeshbach statistical model is used to extrapolate the rates to very high temperatures (i.e. higher relevant astrophysical energies). In this case, the calculations are made with the TALYS code (Goriely et al. 2008).

For each individual reaction, recommended as well as upper and lower limits of the reaction rates are given. Uncertainties in the reaction rates are obtained by modifying the optimal model parameters and still allowing for acceptable fits to the experimental reaction data. All details can be found in $\mathrm{Xu}$ et al. (2011). The improved theoretical treatment compared to NACRE makes the rates, as well as their respective uncertainty estimates, more reliable, especially at low temperatures.

\subsection{The TALYS code}

The neutron, proton, deuterium, tritium, ${ }^{3} \mathrm{He}$ and $\alpha$-particle capture cross sections are, in a first approximation, estimated with the TALYS nuclear reaction code (Koning et al. 2008; Goriely et al. 2008) when not available experimentally or in any other existing compilation of reaction rates. For targets and energies of interest in the present work, TALYS essentially takes the compound mechanisms into account to estimate the total reaction probability, as well as the competition between the various open channels. The cross sections are estimated up to the relevant energies and out of it the Maxwellian-averaged reaction rates for the thermalized target. The calculation includes in the entrance as well as exit channels all single particles of interest here (neutron, proton, deuterium, tritium, ${ }^{3} \mathrm{He}$ and $\alpha$-particle). In the exit channel, multi-particle emission is also taken into account. All the experimental information on nuclear masses, deformation, and low-lying states spectra (including $\gamma$-ray intensities) is considered, whenever available. If not, global nuclear level formulas, $\gamma$-ray strength functions, and nucleon and $\alpha$-particle optical model potentials are considered to determine the excitation level scheme and the photon and particle transmission coefficients.

Based on the statistical model of HauserFeshbach, TALYS is known to be a well-adapted code for medium-mass and heavy target nuclei. Such a model makes the fundamental assumption that the capture process takes place with the intermediary formation of a compound nucleus in thermodynamic equilibrium. The energy of the incident particle is then shared more or less uniformly by all the nucleons before releasing the energy by particle emission or $\gamma$-de-excitation. The formation of a compound nucleus is usually justified by assuming that the level density in the compound nucleus at the projectile incident energy is large enough to ensure an average statistical continuum superposition of available resonances. However, when the number of available states in the compound system is relatively small, as for light targets, the validity of the HauserFeshbach predictions has to be questioned. In this case, other approaches, such a the radiative direct capture within the potential model, need to be considered.

In the present work, the TALYS estimates are considered as a first guess for the reaction rates on light nuclei in order to test the sensitivity of the abundance calculations with respect to changes in the nuclear reaction rates. As shown in Figs. 1,8 the agreement between TALYS and experimental rates are surprisingly good in most of the cases, although most of these reactions should not be physically described by the compound nucleus reaction mechanism. In some of these cases, e.g. ${ }^{9} \mathrm{Be}(\mathrm{p}, \alpha){ }^{6} \mathrm{Li}$, a few resonances are available in the compound nucleus and the predictions are rather satisfactory. In other cases, ${ }^{6} \mathrm{Li}(\mathrm{p}, \gamma){ }^{7} \mathrm{Be}$, no resonances is available and the model fails by about 3 orders of magnitude. 
In contrast, large deviations can be found between TALYS rates and those estimated by Thomas et al. (1993, 1994), as shown in Figs. 9. 11. Even the temperature dependence appears to be quite different1 . In Thomas et al. (1994), in several cases, the same rate is used for the $(\mathrm{p}, \mathrm{n})$, $(\mathrm{p}, \alpha)$ and $(\mathrm{p}, \gamma)$ reaction channels on a given target: an obvious source of errors that can affect the predictions by a few orders of magnitude.

As summarized by the 36 cases studied in Figs. 1] TALYS can globally be expected to provide predictions within 3 orders of magnitude in the temperature range of interest here. Hence variations of these theoretical rates by three orders of magnitude can in a first step be used in our sensitivity analysis for the BBN abundance calculation. A detailed description and study will then be limited to the reactions affecting the abundance predictions in a significant way.

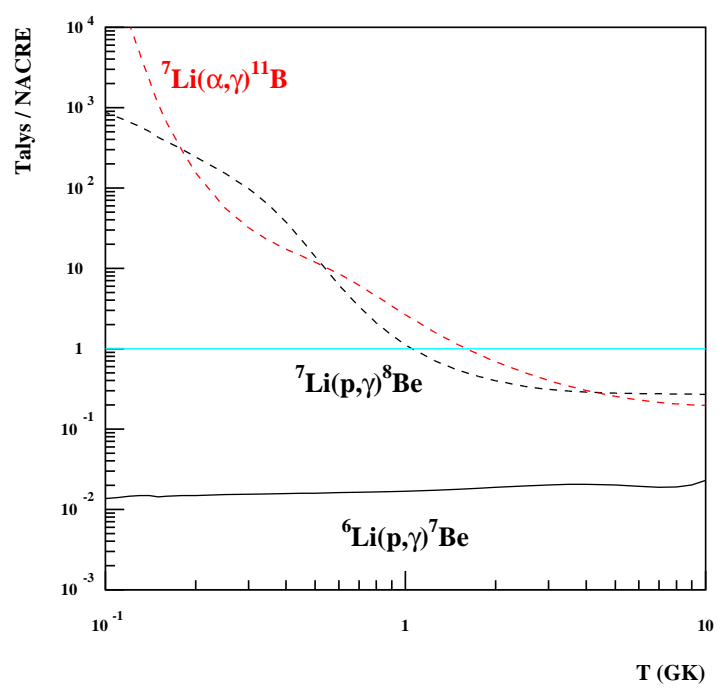

Fig. 1.- Comparison between TALYS and NACRE rates (Angulo et al. 1999).

${ }^{1}$ For the ${ }^{9} \mathrm{Li}(\mathrm{d}, \mathrm{n}){ }^{10}$ Be reaction, this is most probably due to a typo in Thomas et al. (1993) as possibly corrected in the dashed curve in Fig. 9

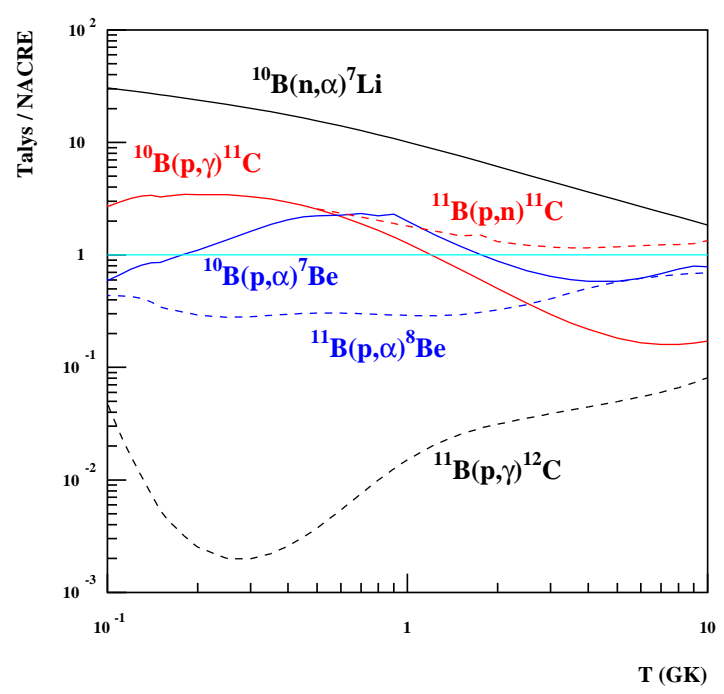

Fig. 2.- Same as Fig. 1

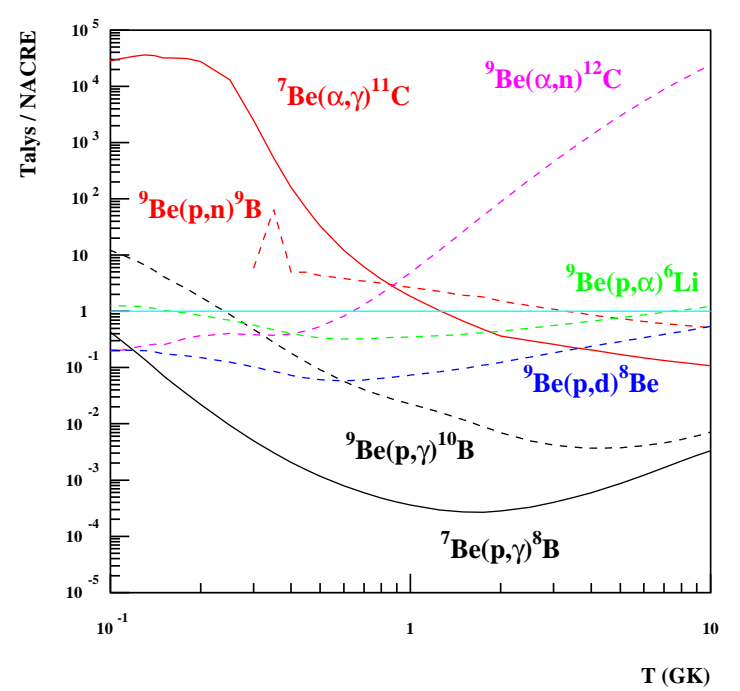

Fig. 3.- Same as Fig. 1 


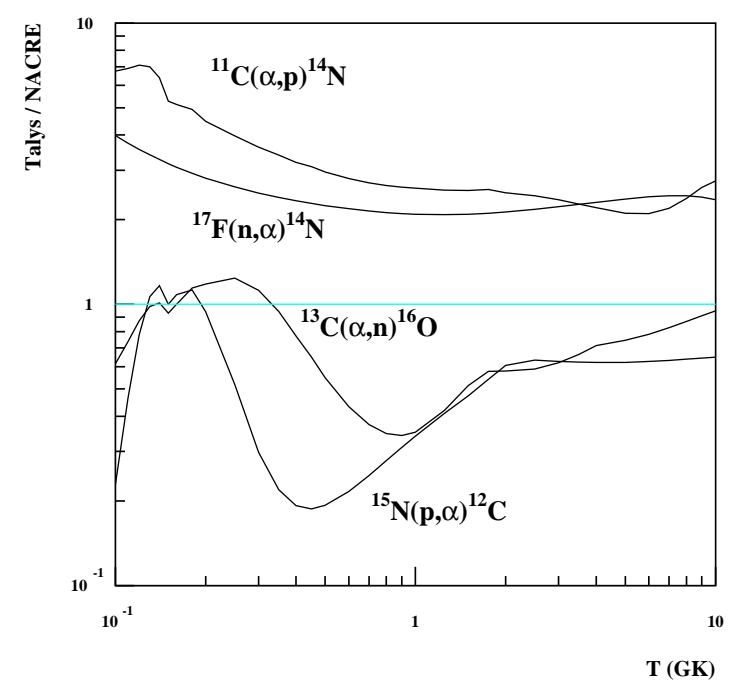

Fig. 4.- Same as Fig. 1

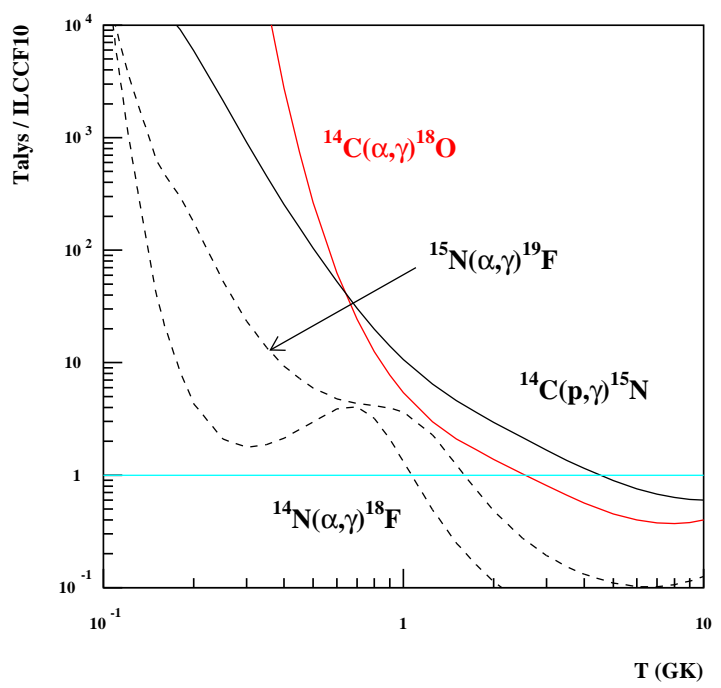

Fig. 5.- Comparison between TALYS and Iliadis et al. (2010).

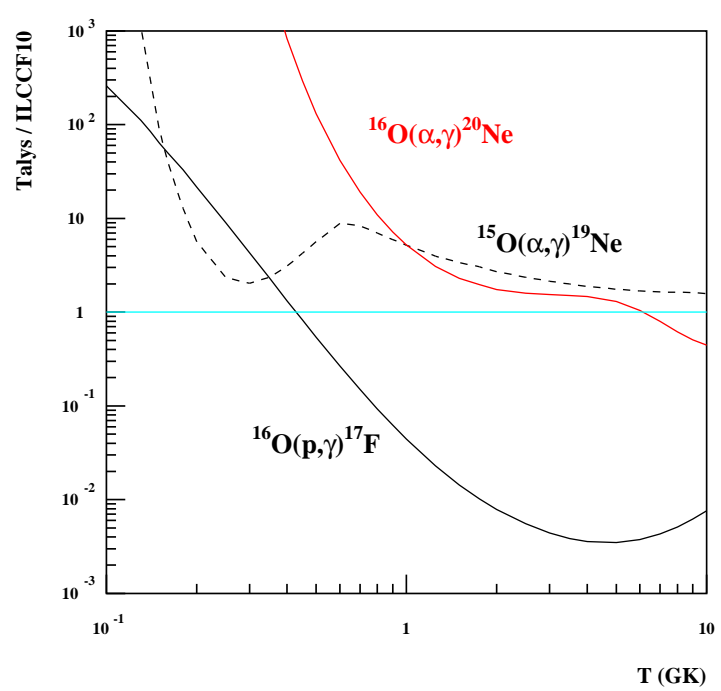

Fig. 6.- Same as Fig. 5

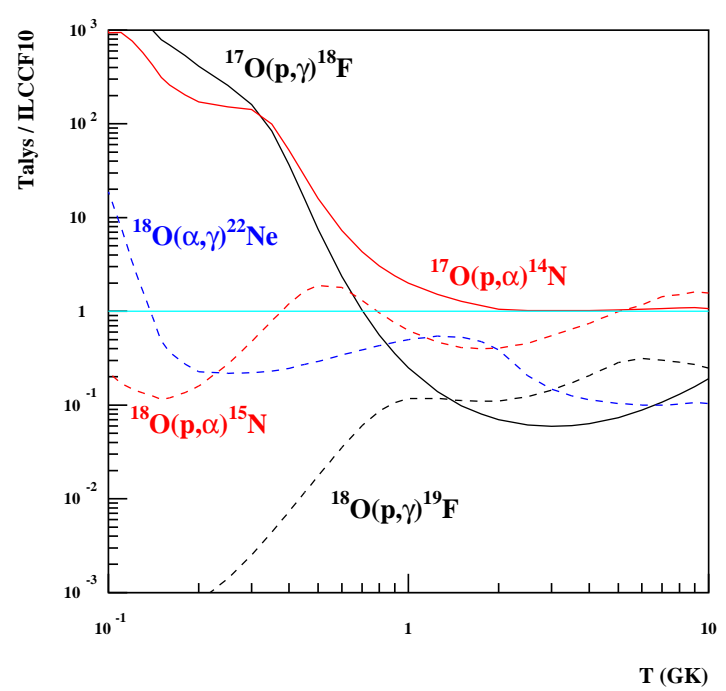

Fig. 7.- Same as Fig. 5 


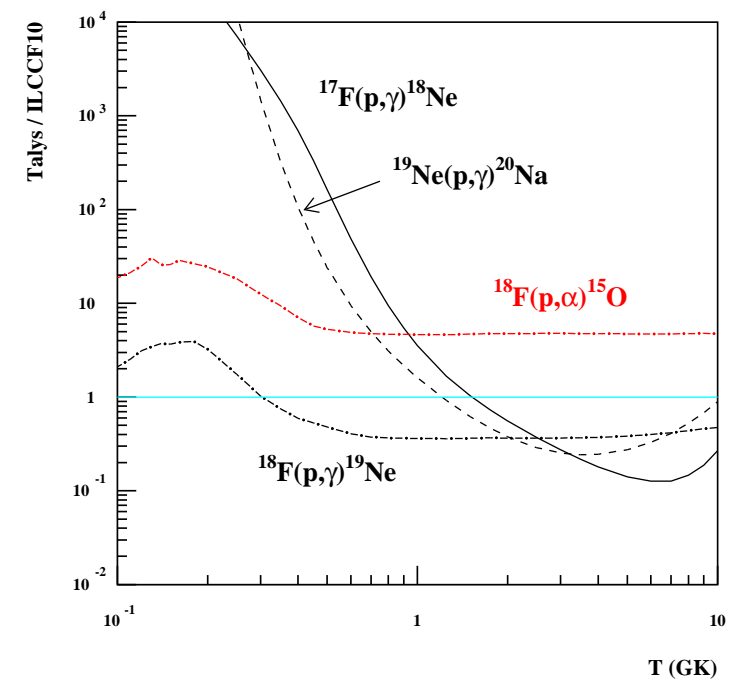

Fig. 8.- Same as Fig. 5

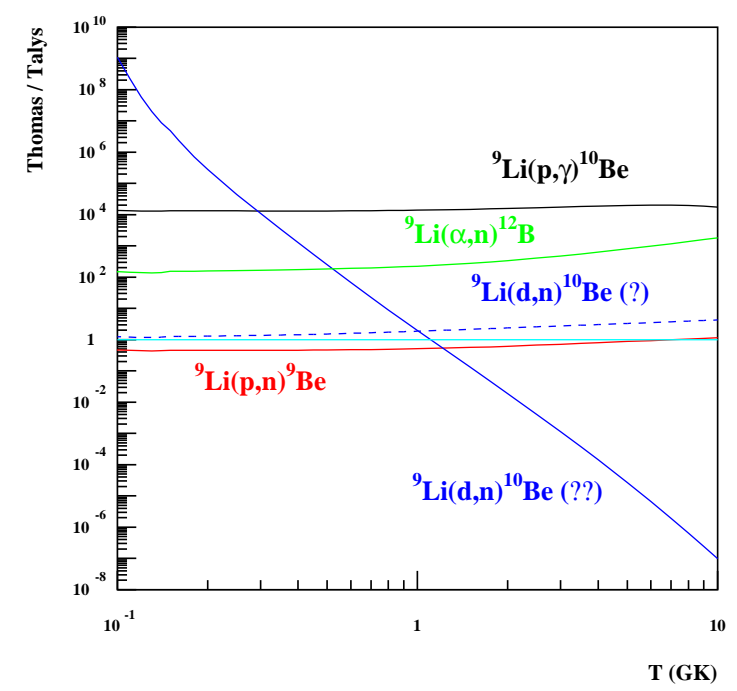

Fig. 9.- Comparison between TALYS and Thomas et al. (1993, 1994)

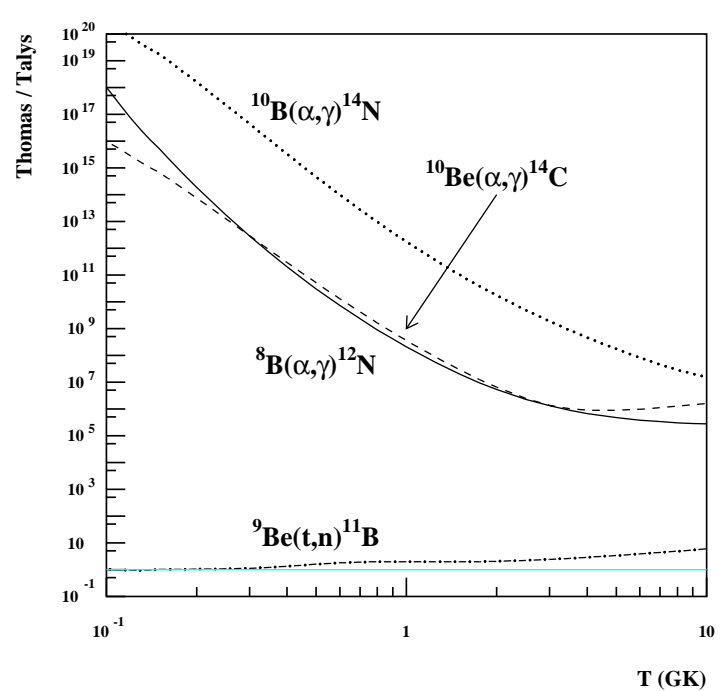

Fig. 10. - Same as Fig. 9

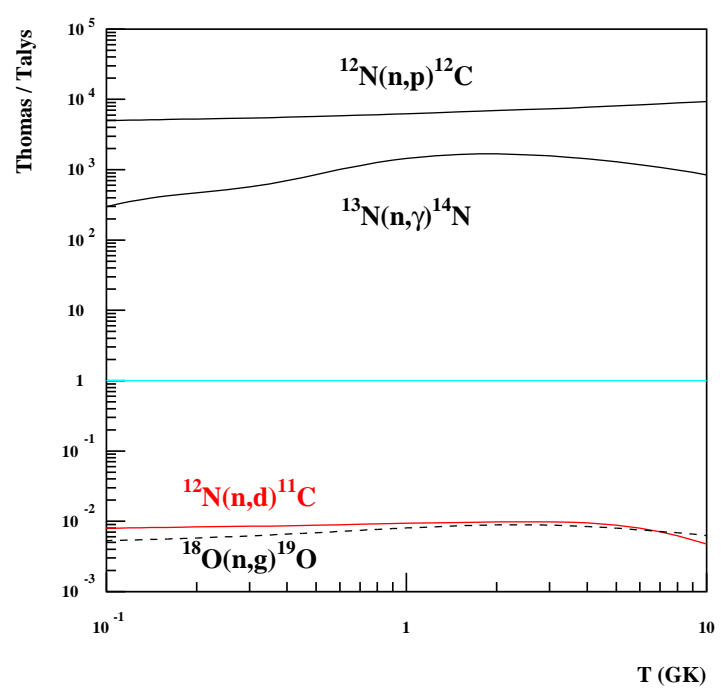

Fig. 11. - Same as Fig. 9 


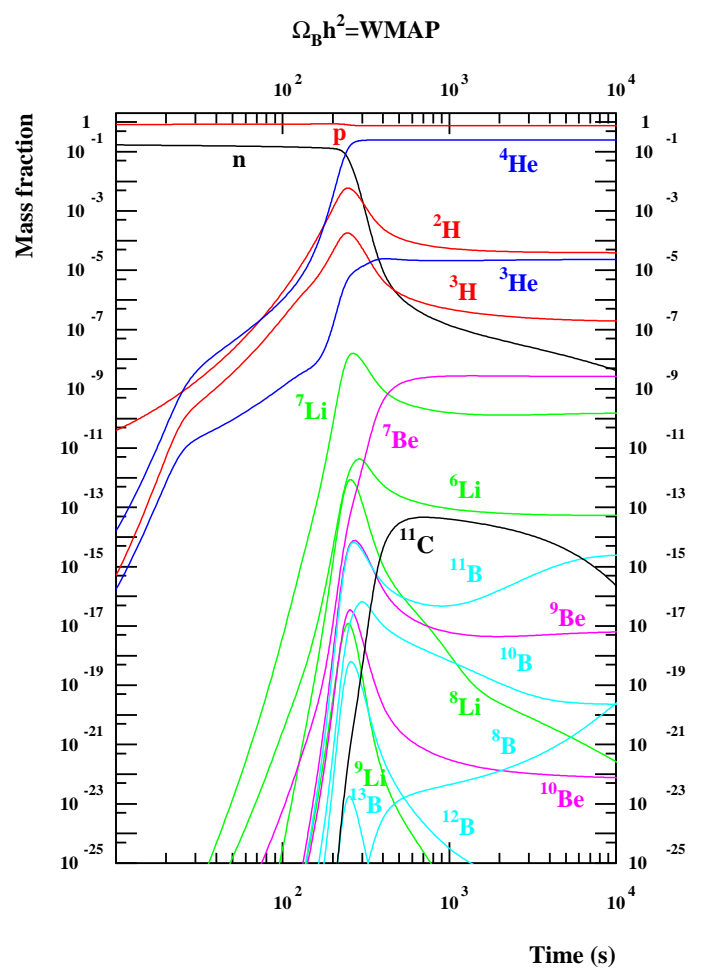

Fig. 12.- (Color online) Standard Big-Bang Nucleosynthesis production of $\mathrm{H}, \mathrm{He}, \mathrm{Li}, \mathrm{Be}$ and $\mathrm{B}$ isotopes as a function of time, for the baryon density taken from WMAP7.

\section{Important reactions for Standard Big- Bang Nucleosynthesis up to CNO}

\subsection{Sensitivity study}

Sensitivity studies have already been performed for the 12 main Standard Big-Bang Nucleosynthesis reactions (Nollett \& Burles 2000; Serpico et al. 2004; Cyburt \& Davids 2008; Coc \& Vangioni 2010) and the many others involved in ${ }^{4} \mathrm{He}, \mathrm{D}$, ${ }^{3} \mathrm{He}$ and ${ }^{7} \mathrm{Li}$ production (Coc et al. 2004). Here, we will consider the impact on ${ }^{9} \mathrm{Be},{ }^{10} \mathrm{~B},{ }^{11} \mathrm{~B}$ and $\mathrm{C}, \mathrm{N}$ and $\mathrm{O}$ isotopes.

In Table 4, we list all reactions included in our network. Our sensitivity study excludes the reactions whose impacts have already been studied in previous work and whose rate uncertainties are documented in Angulo et al. (1999); Descouvemont et al. (2004); Iliadis et al. (2010); Xu et al. (2010). Beta decays are also ex-

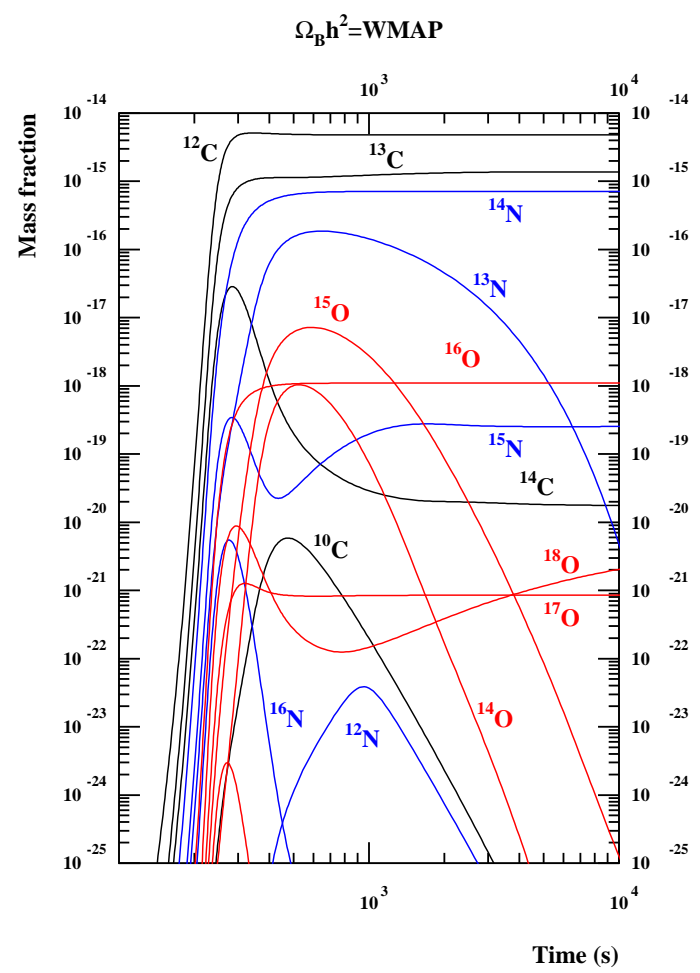

Fig. 13.- (Color online) Standard Big-Bang Nucleosynthesis production of $\mathrm{C}, \mathrm{N}$ and $\mathrm{O}$ isotopes as a function of time. (Note the different time and abundance ranges compared to Fig. 12.)

cluded because all lifetimes are precisely known (Audi et al. 2003). The sensitivity study is performed for each of the 271 reactions whose rates are obtained from TALYS and for those whose uncertainties have not been estimated. Based on the comparison of Section 2.2 between TALYS calculated rates on the one hand and experimental rates on the other hand we consider at most three orders of magnitude variations around the standard reaction rates. More precisely, to estimate the impact of the reaction rates uncertainties on Standard Big-Bang Nucleosynthesis we perform six calculations by changing for each reaction its rate by factors of $0.001,0.01,0.1,10,100$ and 1000 and calculate the relative change in isotopic abundances. Table 1, displays, for each reaction, isotopes for which the relative changes are larger than 20\%. Mass fractions of isotopes with $\mathrm{A} \geq 12$ are added together into CNO. The last column of Table 1 contains either the reference for the origin 


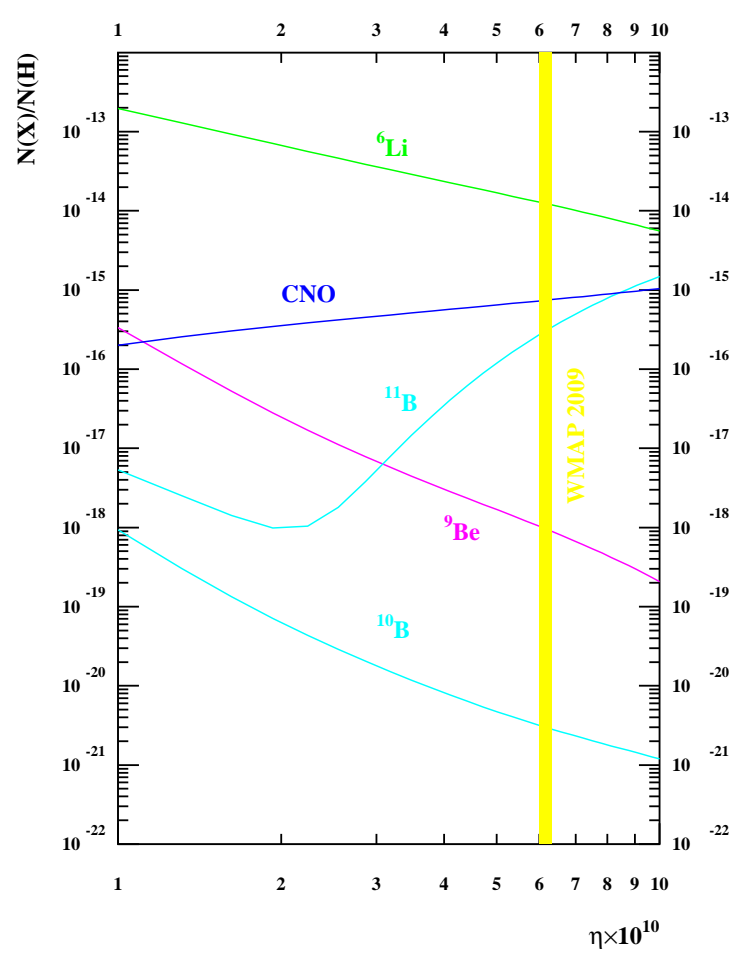

Fig. 14.- (Color online) Standard Big-Bang Nucleosynthesis production of ${ }^{6} \mathrm{Li}, \mathrm{Be}, \mathrm{B}$ and $\mathrm{CNO}$ isotopes as a function of baryonic density.

of the reaction rate or the standard isotopic mass fraction value obtained with the standard values of the reaction rates. In many cases, even if the rate uncertainties have not been explicitly calculated, it is clear that the error bars are much lower than the three orders of magnitudes assumed here, essentially because direct or indirect experimental information exists and may constrain the cross section. However, to keep the procedure simple, we postpone this discussion to the next section. There is indeed little interest to look for accurate estimates of rate uncertainties for reactions which happen to have no impact on the Standard Big-Bang Nucleosynthesis in our sensitivity study. 
TABLE 1

LIST OF REACTIONS INCLUDED IN THE SENSITIVITY TEST WITH REFERENCES FOR THE CORRESPONDING REACtion RATE AND RElative Change in isotopic ABUndanCES WHEN Significant. (SEe teXt.)

\begin{tabular}{|c|c|c|c|c|c|c|c|}
\hline & \multicolumn{6}{|c|}{ Reaction } & Ref. \\
\hline$i$ & \multicolumn{6}{|c|}{ Enhancement factor $X_{i} / X_{i}^{0}$} & $X_{i}^{0}$ \\
\hline \multirow[t]{2}{*}{ Factors } & 0.001 & 0.01 & 0.1 & 10. & 100. & 1000. & $x<\sigma v>$ \\
\hline & \multicolumn{6}{|c|}{$\mathrm{He} 3(\mathrm{t}, \mathrm{np}) \mathrm{He} 4$} & CF88 \\
\hline${ }^{7} \mathrm{Be}$ & $1.00 \times 10^{0}$ & $1.00 \times 10^{0}$ & $1.00 \times 10^{0}$ & $9.97 \times 10^{-1}$ & $9.72 \times 10^{-1}$ & $7.96 \times 10^{-1}$ & $2.61 \times 10^{-9}$ \\
\hline${ }^{8} \mathrm{~B}$ & $1.00 \times 10^{0}$ & $1.00 \times 10^{0}$ & $1.00 \times 10^{0}$ & $9.97 \times 10^{-1}$ & $9.72 \times 10^{-1}$ & $7.96 \times 10^{-1}$ & $1.99 \times 10^{-20}$ \\
\hline${ }^{11} \mathrm{~B}$ & $9.99 \times 10^{-1}$ & $1.00 \times 10^{0}$ & $1.00 \times 10^{0}$ & $9.98 \times 10^{-1}$ & $9.72 \times 10^{-1}$ & $7.90 \times 10^{-1}$ & $4.16 \times 10^{-15}$ \\
\hline \multirow[t]{2}{*}{${ }^{11} \mathrm{C}$} & $1.00 \times 10^{0}$ & $1.00 \times 10^{0}$ & $1.00 \times 10^{0}$ & $9.97 \times 10^{-1}$ & $9.72 \times 10^{-1}$ & $7.89 \times 10^{-1}$ & $8.41 \times 10^{-18}$ \\
\hline & \multicolumn{6}{|c|}{ He3(t, $\gamma) \mathrm{Li} 6$} & FK90 \\
\hline${ }^{6} \mathrm{Li}$ & $9.97 \times 10^{-1}$ & $9.97 \times 10^{-1}$ & $9.97 \times 10^{-1}$ & $1.03 \times 10^{0}$ & $1.31 \times 10^{0}$ & $4.11 \times 10^{0}$ & $5.56 \times 10^{-14}$ \\
\hline \multirow[t]{2}{*}{${ }^{10} \mathrm{~B}$} & $9.98 \times 10^{-1}$ & $9.98 \times 10^{-1}$ & $9.98 \times 10^{-1}$ & $1.02 \times 10^{0}$ & $1.22 \times 10^{0}$ & $3.20 \times 10^{0}$ & $2.16 \times 10^{-20}$ \\
\hline & \multicolumn{6}{|c|}{ He4 $(\mathrm{d}, \gamma) \operatorname{Li} 6$} & Ham10 \\
\hline${ }^{6} \mathrm{Li}$ & $4.12 \times 10^{-3}$ & $1.31 \times 10^{-2}$ & $1.03 \times 10^{-1}$ & $9.97 \times 10^{0}$ & $9.97 \times 10^{1}$ & $9.95 \times 10^{2}$ & $5.56 \times 10^{-14}$ \\
\hline \multirow[t]{2}{*}{${ }^{10} \mathrm{~B}$} & $4.43 \times 10^{-1}$ & $4.48 \times 10^{-1}$ & $4.98 \times 10^{-1}$ & $6.02 \times 10^{0}$ & $5.62 \times 10^{1}$ & $5.57 \times 10^{2}$ & $2.16 \times 10^{-20}$ \\
\hline & \multicolumn{6}{|c|}{$\overline{\operatorname{Li} 6(\alpha, \gamma) \mathrm{B} 10}$} & CF88 \\
\hline \multirow[t]{2}{*}{${ }^{10} \mathrm{~B}$} & $4.41 \times 10^{-1}$ & $4.46 \times 10^{-1}$ & $4.96 \times 10^{-1}$ & $6.04 \times 10^{0}$ & $5.64 \times 10^{1}$ & $5.60 \times 10^{2}$ & $2.16 \times 10^{-20}$ \\
\hline & \multicolumn{6}{|c|}{$\operatorname{Li7}(n, \gamma) \operatorname{Li} 8$} & MF89Hei98 \\
\hline \multirow[t]{2}{*}{${ }^{8} \mathrm{Li}$} & $1.03 \times 10^{-3}$ & $1.00 \times 10^{-2}$ & $1.00 \times 10^{-1}$ & $1.00 \times 10^{1}$ & $1.00 \times 10^{2}$ & $1.00 \times 10^{3}$ & $4.24 \times 10^{-23}$ \\
\hline & \multicolumn{6}{|c|}{$\operatorname{Li} 7(\mathrm{~d}, \gamma) \mathrm{Be} 9$} & TALYS $\dagger$ \\
\hline${ }^{9} \mathrm{Be}$ & $8.31 \times 10^{-1}$ & $8.33 \times 10^{-1}$ & $8.48 \times 10^{-1}$ & $2.52 \times 10^{0}$ & $1.77 \times 10^{1}$ & $1.70 \times 10^{2}$ & $2.90 \times 10^{-18}$ \\
\hline${ }^{10} \mathrm{Be}$ & $1.00 \times 10^{0}$ & $1.00 \times 10^{0}$ & $1.00 \times 10^{0}$ & $1.00 \times 10^{0}$ & $1.04 \times 10^{0}$ & $1.42 \times 10^{0}$ & $7.32 \times 10^{-23}$ \\
\hline${ }^{10} \mathrm{~B}$ & $9.90 \times 10^{-1}$ & $9.90 \times 10^{-1}$ & $9.91 \times 10^{-1}$ & $1.09 \times 10^{0}$ & $2.03 \times 10^{0}$ & $1.14 \times 10^{1}$ & $2.16 \times 10^{-20}$ \\
\hline \multirow[t]{2}{*}{$\mathrm{CNO}$} & $9.99 \times 10^{-1}$ & $9.99 \times 10^{-1}$ & $9.99 \times 10^{-1}$ & $1.01 \times 10^{0}$ & $1.11 \times 10^{0}$ & $2.10 \times 10^{0}$ & $4.98 \times 10^{-15}$ \\
\hline & \multicolumn{6}{|c|}{ Li7(d,n)2He4 } & Boyd93† \\
\hline${ }^{10} \mathrm{Be}$ & $1.00 \times 10^{0}$ & $1.00 \times 10^{0}$ & $1.00 \times 10^{0}$ & $9.98 \times 10^{-1}$ & $9.72 \times 10^{-1}$ & $7.93 \times 10^{-1}$ & $7.32 \times 10^{-23}$ \\
\hline \multirow[t]{2}{*}{$\mathrm{CNO}$} & $1.66 \times 10^{0}$ & $1.65 \times 10^{0}$ & $1.55 \times 10^{0}$ & $2.80 \times 10^{-1}$ & $5.99 \times 10^{-2}$ & $2.18 \times 10^{-2}$ & $4.98 \times 10^{-15}$ \\
\hline & \multicolumn{6}{|c|}{$\operatorname{Li7}(\mathrm{t}, \gamma) \operatorname{Be} 10$} & TALYS \\
\hline \multirow[t]{2}{*}{${ }^{10} \mathrm{Be}$} & $3.89 \times 10^{-3}$ & $1.29 \times 10^{-2}$ & $1.03 \times 10^{-1}$ & $9.97 \times 10^{0}$ & $9.97 \times 10^{1}$ & $9.97 \times 10^{2}$ & $7.32 \times 10^{-23}$ \\
\hline & \multicolumn{6}{|c|}{ Li7(t,n)Be9 } & $\overline{\text { Bru90† }}$ \\
\hline${ }^{9} \mathrm{Be}$ & $5.24 \times 10^{-1}$ & $5.28 \times 10^{-1}$ & $5.71 \times 10^{-1}$ & $5.29 \times 10^{0}$ & $4.82 \times 10^{1}$ & $4.77 \times 10^{2}$ & $2.90 \times 10^{-18}$ \\
\hline${ }^{10} \mathrm{Be}$ & $9.99 \times 10^{-1}$ & $9.99 \times 10^{-1}$ & $9.99 \times 10^{-1}$ & $1.01 \times 10^{0}$ & $1.12 \times 10^{0}$ & $2.23 \times 10^{0}$ & $7.32 \times 10^{-23}$ \\
\hline${ }^{10} \mathrm{~B}$ & $9.67 \times 10^{-1}$ & $9.67 \times 10^{-1}$ & $9.70 \times 10^{-1}$ & $1.30 \times 10^{0}$ & $4.29 \times 10^{0}$ & $3.42 \times 10^{1}$ & $2.16 \times 10^{-20}$ \\
\hline \multirow[t]{2}{*}{$\mathrm{CNO}$} & $9.88 \times 10^{-1}$ & $9.89 \times 10^{-1}$ & $9.90 \times 10^{-1}$ & $1.10 \times 10^{0}$ & $2.14 \times 10^{0}$ & $1.17 \times 10^{1}$ & $4.98 \times 10^{-15}$ \\
\hline & \multicolumn{6}{|c|}{$\mathrm{Li} 7(\mathrm{t}, 2 \mathrm{n}) 2 \mathrm{He} 4$} & CF88\&MF89 \\
\hline \multirow[t]{2}{*}{$\mathrm{CNO}$} & $1.00 \times 10^{0}$ & $1.00 \times 10^{0}$ & $1.00 \times 10^{0}$ & $9.91 \times 10^{-1}$ & $9.13 \times 10^{-1}$ & $5.32 \times 10^{-1}$ & $4.98 \times 10^{-15}$ \\
\hline & \multicolumn{6}{|c|}{ 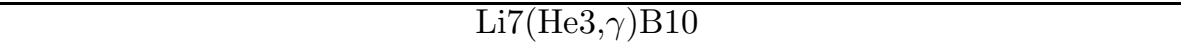 } & TALYS \\
\hline \multirow[t]{2}{*}{${ }^{10} \mathrm{~B}$} & $9.93 \times 10^{-1}$ & $9.93 \times 10^{-1}$ & $9.94 \times 10^{-1}$ & $1.06 \times 10^{0}$ & $1.70 \times 10^{0}$ & $8.08 \times 10^{0}$ & $2.16 \times 10^{-20}$ \\
\hline & & & $\mathrm{Li7}(\mathrm{H}$ & ,p)Be9 & & & TALYS \\
\hline${ }^{9} \mathrm{Be}$ & $9.96 \times 10^{-1}$ & $9.96 \times 10^{-1}$ & $9.96 \times 10^{-1}$ & $1.04 \times 10^{0}$ & $1.45 \times 10^{0}$ & $5.49 \times 10^{0}$ & $2.90 \times 10^{-18}$ \\
\hline${ }^{10} \mathrm{~B}$ & $9.98 \times 10^{-1}$ & $9.98 \times 10^{-1}$ & $9.98 \times 10^{-1}$ & $1.02 \times 10^{0}$ & $1.19 \times 10^{0}$ & $2.92 \times 10^{0}$ & $2.16 \times 10^{-20}$ \\
\hline
\end{tabular}


TABLE 1-Continued

\begin{tabular}{|c|c|c|c|c|c|c|c|}
\hline & \multicolumn{6}{|c|}{ Li8(n, $\gamma)$ Li9 } & Rau94 \\
\hline \multirow[t]{2}{*}{$\mathrm{CNO}$} & $9.99 \times 10^{-1}$ & $9.99 \times 10^{-1}$ & $9.99 \times 10^{-1}$ & $1.01 \times 10^{0}$ & $1.06 \times 10^{0}$ & $1.62 \times 10^{0}$ & $4.98 \times 10^{-15}$ \\
\hline & \multicolumn{6}{|c|}{ Li8(t,n)Be10 } & TALYS \\
\hline \multirow[t]{2}{*}{$\mathrm{CNO}$} & $1.00 \times 10^{0}$ & $1.00 \times 10^{0}$ & $1.00 \times 10^{0}$ & $1.00 \times 10^{0}$ & $1.02 \times 10^{0}$ & $1.23 \times 10^{0}$ & $4.98 \times 10^{-15}$ \\
\hline & \multicolumn{6}{|c|}{$\operatorname{Li} 8(\alpha, \gamma) \mathrm{B} 12$} & TALYS \\
\hline \multirow{2}{*}{$\mathrm{CNO}$} & $9.99 \times 10^{-1}$ & $9.99 \times 10^{-1}$ & $9.99 \times 10^{-1}$ & $1.01 \times 10^{0}$ & $1.11 \times 10^{0}$ & $2.15 \times 10^{0}$ & $4.98 \times 10^{-15}$ \\
\hline & \multicolumn{6}{|c|}{$\operatorname{Li} 8(\alpha, n) B 11$} & $\overline{M i z 01 \dagger}$ \\
\hline \multirow[t]{2}{*}{$\mathrm{CNO}$} & $8.92 \times 10^{-1}$ & $8.93 \times 10^{-1}$ & $9.03 \times 10^{-1}$ & $1.97 \times 10^{0}$ & $1.12 \times 10^{1}$ & $7.81 \times 10^{1}$ & $4.98 \times 10^{-15}$ \\
\hline & \multicolumn{6}{|c|}{$\operatorname{Li} 9(\alpha, \mathrm{n}) \mathrm{B} 12$} & TALYS \\
\hline \multirow[t]{2}{*}{$\mathrm{CNO}$} & $9.99 \times 10^{-1}$ & $9.99 \times 10^{-1}$ & $9.99 \times 10^{-1}$ & $1.01 \times 10^{0}$ & $1.08 \times 10^{0}$ & $1.73 \times 10^{0}$ & $4.98 \times 10^{-15}$ \\
\hline & \multicolumn{6}{|c|}{$\mathrm{Be} 7(\mathrm{~d}, \mathrm{p}) 2 \mathrm{He} 4$} & CF88 \\
\hline${ }^{7} \mathrm{Li}$ & $1.01 \times 10^{0}$ & $1.01 \times 10^{0}$ & $1.00 \times 10^{0}$ & $9.57 \times 10^{-1}$ & $7.36 \times 10^{-1}$ & $5.05 \times 10^{-1}$ & $1.54 \times 10^{-10}$ \\
\hline${ }^{8} \mathrm{Li}$ & $1.01 \times 10^{0}$ & $1.01 \times 10^{0}$ & $1.00 \times 10^{0}$ & $9.57 \times 10^{-1}$ & $7.36 \times 10^{-1}$ & $5.05 \times 10^{-1}$ & $4.24 \times 10^{-23}$ \\
\hline${ }^{7} \mathrm{Be}$ & $1.01 \times 10^{0}$ & $1.01 \times 10^{0}$ & $1.01 \times 10^{0}$ & $9.23 \times 10^{-1}$ & $5.27 \times 10^{-1}$ & $1.13 \times 10^{-1}$ & $2.61 \times 10^{-9}$ \\
\hline${ }^{9} \mathrm{Be}$ & $1.01 \times 10^{0}$ & $1.01 \times 10^{0}$ & $1.01 \times 10^{0}$ & $9.46 \times 10^{-1}$ & $6.67 \times 10^{-1}$ & $3.77 \times 10^{-1}$ & $2.90 \times 10^{-18}$ \\
\hline${ }^{10} \mathrm{Be}$ & $1.01 \times 10^{0}$ & $1.00 \times 10^{0}$ & $1.00 \times 10^{0}$ & $9.58 \times 10^{-1}$ & $7.43 \times 10^{-1}$ & $5.19 \times 10^{-1}$ & $7.32 \times 10^{-23}$ \\
\hline${ }^{8} \mathrm{~B}$ & $1.01 \times 10^{0}$ & $1.01 \times 10^{0}$ & $1.01 \times 10^{0}$ & $9.23 \times 10^{-1}$ & $5.27 \times 10^{-1}$ & $1.14 \times 10^{-1}$ & $1.99 \times 10^{-20}$ \\
\hline${ }^{10} \mathrm{~B}$ & $1.00 \times 10^{0}$ & $1.00 \times 10^{0}$ & $1.00 \times 10^{0}$ & $9.68 \times 10^{-1}$ & $8.05 \times 10^{-1}$ & $6.34 \times 10^{-1}$ & $2.16 \times 10^{-20}$ \\
\hline${ }^{11} \mathrm{~B}$ & $1.01 \times 10^{0}$ & $1.01 \times 10^{0}$ & $1.01 \times 10^{0}$ & $9.32 \times 10^{-1}$ & $5.49 \times 10^{-1}$ & $1.06 \times 10^{-1}$ & $4.16 \times 10^{-15}$ \\
\hline \multirow[t]{2}{*}{${ }^{11} \mathrm{C}$} & $1.01 \times 10^{0}$ & $1.01 \times 10^{0}$ & $1.01 \times 10^{0}$ & $9.32 \times 10^{-1}$ & $5.49 \times 10^{-1}$ & $1.06 \times 10^{-1}$ & $8.41 \times 10^{-18}$ \\
\hline & \multicolumn{6}{|c|}{$\mathrm{Be} 7(\mathrm{t}, \gamma) \mathrm{B} 10$} & TALYS \\
\hline \multirow[t]{2}{*}{${ }^{10} \mathrm{~B}$} & $6.81 \times 10^{-1}$ & $6.84 \times 10^{-1}$ & $7.13 \times 10^{-1}$ & $3.87 \times 10^{0}$ & $3.26 \times 10^{1}$ & $3.20 \times 10^{2}$ & $2.16 \times 10^{-20}$ \\
\hline & \multicolumn{6}{|c|}{ Be7(t,p)Be9 } & TALYS † \\
\hline${ }^{9} \mathrm{Be}$ & $6.51 \times 10^{-1}$ & $6.54 \times 10^{-1}$ & $6.85 \times 10^{-1}$ & $4.15 \times 10^{0}$ & $3.56 \times 10^{1}$ & $3.45 \times 10^{2}$ & $2.90 \times 10^{-18}$ \\
\hline${ }^{10} \mathrm{Be}$ & $9.99 \times 10^{-1}$ & $9.99 \times 10^{-1}$ & $9.99 \times 10^{-1}$ & $1.01 \times 10^{0}$ & $1.11 \times 10^{0}$ & $2.14 \times 10^{0}$ & $7.32 \times 10^{-23}$ \\
\hline \multirow[t]{2}{*}{${ }^{10} \mathrm{~B}$} & $9.32 \times 10^{-1}$ & $9.33 \times 10^{-1}$ & $9.39 \times 10^{-1}$ & $1.61 \times 10^{0}$ & $7.72 \times 10^{0}$ & $6.79 \times 10^{1}$ & $2.16 \times 10^{-20}$ \\
\hline & \multicolumn{6}{|c|}{$\overline{B e} 9(d, n) B 10$} & TALYS \\
\hline \multirow[t]{2}{*}{${ }^{10} \mathrm{~B}$} & $9.98 \times 10^{-1}$ & $9.98 \times 10^{-1}$ & $9.98 \times 10^{-1}$ & $1.02 \times 10^{0}$ & $1.23 \times 10^{0}$ & $3.33 \times 10^{0}$ & $2.16 \times 10^{-20}$ \\
\hline & \multicolumn{6}{|c|}{ Be9(d,p)Be10 } & TALYS \\
\hline \multirow[t]{2}{*}{${ }^{10} \mathrm{Be}$} & $9.97 \times 10^{-1}$ & $9.97 \times 10^{-1}$ & $9.97 \times 10^{-1}$ & $1.03 \times 10^{0}$ & $1.28 \times 10^{0}$ & $3.78 \times 10^{0}$ & $7.32 \times 10^{-23}$ \\
\hline & \multicolumn{6}{|c|}{$\operatorname{Be10}(\mathrm{p}, \alpha) \operatorname{Li} 7$} & TALYS \\
\hline \multirow[t]{2}{*}{${ }^{10} \mathrm{Be}$} & $6.18 \times 10^{2}$ & $1.53 \times 10^{2}$ & $1.23 \times 10^{1}$ & $8.70 \times 10^{-2}$ & $8.23 \times 10^{-3}$ & $8.13 \times 10^{-4}$ & $7.32 \times 10^{-23}$ \\
\hline & \multicolumn{6}{|c|}{$\overline{\operatorname{Be} 10}(\alpha, \mathrm{n}) \mathrm{C} 13$} & TALYS \\
\hline \multirow[t]{2}{*}{$\mathrm{CNO}$} & $1.00 \times 10^{0}$ & $1.00 \times 10^{0}$ & $1.00 \times 10^{0}$ & $1.00 \times 10^{0}$ & $1.03 \times 10^{0}$ & $1.28 \times 10^{0}$ & $4.98 \times 10^{-15}$ \\
\hline & & & $\mathrm{B} 11(\mathrm{r}$ & $\gamma) \mathrm{B} 12$ & & & Rau94† \\
\hline $\mathrm{CNO}$ & $9.10 \times 10^{-1}$ & $9.11 \times 10^{-1}$ & $9.19 \times 10^{-1}$ & $1.81 \times 10^{0}$ & $9.91 \times 10^{0}$ & $8.77 \times 10^{1}$ & $4.98 \times 10^{-15}$ \\
\hline & & & $\mathrm{B} 11(\mathrm{c}$ & n)C12 & & & TALYS † \\
\hline $\mathrm{CNO}$ & $7.04 \times 10^{-1}$ & $7.06 \times 10^{-1}$ & $7.33 \times 10^{-1}$ & $3.67 \times 10^{0}$ & $3.02 \times 10^{1}$ & $2.80 \times 10^{2}$ & $4.98 \times 10^{-15}$ \\
\hline & & & B11(c & p)B12 & & & TALYS † \\
\hline $\mathrm{CNO}$ & $9.92 \times 10^{-1}$ & $9.92 \times 10^{-1}$ & $9.92 \times 10^{-1}$ & $1.08 \times 10^{0}$ & $1.83 \times 10^{0}$ & $9.33 \times 10^{0}$ & $4.98 \times 10^{-15}$ \\
\hline & & & $\mathrm{B} 11(1$ & a)C13 & & & TALYS \\
\hline $\mathrm{CNO}$ & $9.99 \times 10^{-1}$ & $9.99 \times 10^{-1}$ & $9.99 \times 10^{-1}$ & $1.01 \times 10^{0}$ & $1.12 \times 10^{0}$ & $2.17 \times 10^{0}$ & $4.98 \times 10^{-15}$ \\
\hline
\end{tabular}


TABLE 1-Continued

\begin{tabular}{|c|c|c|c|c|c|c|c|}
\hline & \multicolumn{6}{|c|}{$\mathrm{C} 11(\mathrm{n}, \gamma) \mathrm{C} 12$} & Rau94 \\
\hline $\mathrm{CNO}$ & $9.99 \times 10^{-1}$ & $9.99 \times 10^{-1}$ & $9.99 \times 10^{-1}$ & $1.01 \times 10^{0}$ & $1.08 \times 10^{0}$ & $1.75 \times 10^{0}$ & $4.98 \times 10^{-15}$ \\
\hline & \multicolumn{6}{|c|}{$\mathrm{C} 11(\mathrm{n}, \alpha) 2 \mathrm{He} 4$} & Rau94† \\
\hline${ }^{11} \mathrm{~B}$ & $1.16 \times 10^{0}$ & $1.16 \times 10^{0}$ & $1.15 \times 10^{0}$ & $4.02 \times 10^{-1}$ & $1.16 \times 10^{-2}$ & $1.63 \times 10^{-4}$ & $4.16 \times 10^{-15}$ \\
\hline${ }^{11} \mathrm{C}$ & $1.16 \times 10^{0}$ & $1.16 \times 10^{0}$ & $1.15 \times 10^{0}$ & $4.01 \times 10^{-1}$ & $1.11 \times 10^{-2}$ & $2.77 \times 10^{-6}$ & $8.41 \times 10^{-18}$ \\
\hline & \multicolumn{6}{|c|}{$\mathrm{C} 11(\mathrm{~d}, \mathrm{p}) \mathrm{C} 12$} & TALYS† \\
\hline $\mathrm{CNO}$ & $9.94 \times 10^{-1}$ & $9.94 \times 10^{-1}$ & $9.95 \times 10^{-1}$ & $1.05 \times 10^{0}$ & $1.55 \times 10^{0}$ & $5.67 \times 10^{0}$ & $4.98 \times 10^{-15}$ \\
\hline & \multicolumn{6}{|c|}{$\mathrm{C} 12(\mathrm{t}, \alpha) \mathrm{B} 11$} & TALYS \\
\hline $\mathrm{CNO}$ & $1.00 \times 10^{0}$ & $1.00 \times 10^{0}$ & $1.00 \times 10^{0}$ & $9.97 \times 10^{-1}$ & $9.68 \times 10^{-1}$ & $7.49 \times 10^{-1}$ & $4.98 \times 10^{-15}$ \\
\hline & \multicolumn{6}{|c|}{$\mathrm{C} 13(\mathrm{~d}, \alpha) \mathrm{B} 11$} & TALYS \\
\hline $\mathrm{CNO}$ & $1.00 \times 10^{0}$ & $1.00 \times 10^{0}$ & $1.00 \times 10^{0}$ & $9.63 \times 10^{-1}$ & $8.42 \times 10^{-1}$ & $7.52 \times 10^{-1}$ & $4.98 \times 10^{-15}$ \\
\hline
\end{tabular}

$\dagger$ Reaction rate re-evaluated in Section 3.2 
The examination of Table 1 shows that only a few reactions have a strong impact on the $\mathrm{CNO}$ or LiBeB productions. We did not consider reactions whose only impact would be on ${ }^{10} \mathrm{~B}$ ( or ${ }^{10} \mathrm{Be}$ ), because its abundance would anyway remains negligible when compared to ${ }^{11} \mathrm{~B}$.

Note that even with a factor $10^{3}$ rate increase we have found no ${ }^{7} \mathrm{Li}$ or ${ }^{7} \mathrm{Be} n-, p-, d-$, ${ }^{3} \mathrm{He}-$ or $\alpha$-induced reactions that would significantly reduce the ${ }^{7} \mathrm{Li}+{ }^{7} \mathrm{Be}$ abundance as suggested by Chakraborty et al. (2011), except for the ${ }^{7} \mathrm{Be}(\mathrm{d}, \mathrm{p})$ reaction already considered by Coc et al. (2004); Angulo et al. (2005); Cyburt \& Pospelov (2009).

Reactions affecting the ${ }^{6} \mathrm{Li}$ nucleosynthesis are ${ }^{4} \mathrm{He}(\mathrm{d}, \gamma){ }^{6} \mathrm{Li}$ and to a much lower extent ${ }^{3} \mathrm{He}(\mathrm{t}, \gamma){ }^{6} \mathrm{Li}$. The former has recently been experimentally re-investigated (Hammache et al. 2010) and its rate uncertainty should not exceed some $40 \%$. The rate of the latter has been calculated by Fukugita \& Kajino (1990) without providing an estimate of the associated uncertainty that should, in any case, be much lower that the factor of 1000 needed.

The ${ }^{9} \mathrm{Be}$ nucleosynthesis is sensitive to the ${ }^{7} \mathrm{Li}(\mathrm{t}, \mathrm{n}){ }^{9}$ Be reaction (Boyd \& Kajino 1989; Brune et al. 1991; Barhoumi et al. 1991) but also to the ${ }^{7} \mathrm{Li}(\mathrm{d}, \gamma){ }^{9} \mathrm{Be},{ }^{7} \mathrm{Be}(\mathrm{t}, \mathrm{p}){ }^{9} \mathrm{Be}$ reactions and to a lower extent to the ${ }^{7} \mathrm{Li}\left({ }^{3} \mathrm{He}, \mathrm{p}\right){ }^{9} \mathrm{Be}$. These rates are discussed in Section 3.2 .

The ${ }^{11} \mathrm{~B}$ production could be drastically reduced if the ${ }^{11} \mathrm{C}(\mathrm{n}, \alpha) 2^{4} \mathrm{He}$ reaction rate (Section 3.2) was higher.

The CNO production is significantly sensitive (more than by a factor of about 2) to several reaction rates. In particular, these include: $\quad{ }^{7} \mathrm{Li}(\mathrm{d}, \mathrm{n}) 2{ }^{4} \mathrm{He},{ }^{7} \mathrm{Li}(\mathrm{t}, \mathrm{n}){ }^{9} \mathrm{Be},{ }^{8} \mathrm{Li}(\alpha, \mathrm{n}){ }^{11} \mathrm{~B}$, ${ }^{11} \mathrm{~B}(\mathrm{n}, \gamma){ }^{12} \mathrm{C},{ }^{11} \mathrm{~B}(\mathrm{~d}, \mathrm{n}){ }^{12} \mathrm{C},{ }^{11} \mathrm{~B}(\mathrm{~d}, \mathrm{p}){ }^{12} \mathrm{~B}$ as well as ${ }^{11} \mathrm{C}(\mathrm{d}, \mathrm{p}){ }^{12} \mathrm{C}$. We re-evaluate their rates in $\mathrm{Sec}-$ tion 3.2. The impact of ${ }^{7} \mathrm{Li}(\mathrm{d}, \mathrm{n}) 2^{4} \mathrm{He}$ is unexpected and should be compared to the influence of ${ }^{1} \mathrm{H}(\mathrm{n}, \gamma)^{2} \mathrm{H}$ on ${ }^{7} \mathrm{Li}$. Indeed Fig. 15 shows the effect of increasing the ${ }^{7} \mathrm{Li}(\mathrm{d}, \mathrm{n}) 2{ }^{4} \mathrm{He}$ reaction rate by a factor of 1000. Even though the final abundances are left unchanged, the peak ${ }^{7} \mathrm{Li}$ abundance at $t \approx 200 \mathrm{~s}$ is reduced by a factor of about 100 . A similar evolution is followed by the ${ }^{8} \mathrm{Li}$ and $\mathrm{CNO}$ abundances (not shown on the figure).

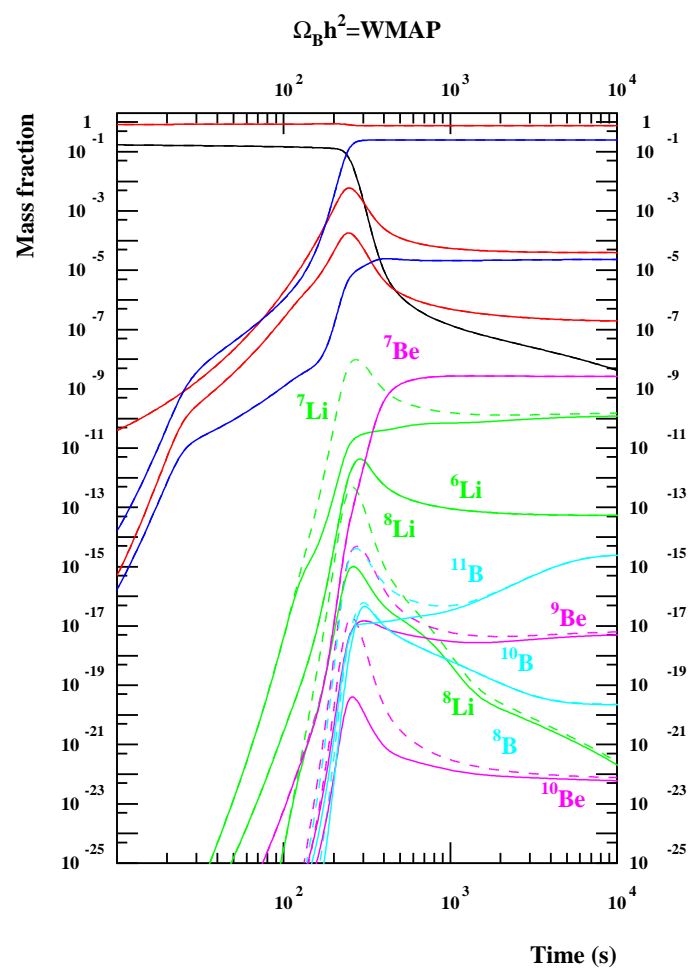

Fig. 15.- (Color online) Standard Big-Bang Nucleosynthesis production of $\mathrm{H}, \mathrm{He}, \mathrm{Li}, \mathrm{Be}$ and $\mathrm{B}$ isotopes with the ${ }^{7} \mathrm{Li}(\mathrm{d}, \mathrm{n}) 2^{4} \mathrm{He}$ reaction rate from Boyd et al. (1993) (dashed lines, corresponding to Fig. 12) and with the same rate multiplied by a factor of 1000 (solid lines).

\subsection{Improvement of some critical reaction rates}

In this Section, the above-mentioned critical reactions are analyzed and their rates re-evaluated on the basis of suited reaction models. In addition, realistic uncertainties affecting these rates are estimated in order to provide realistic predictions for the BBN. Since each reaction represents a specific case dominated by a specific reaction mechanism, they are analyzed and evaluated separately below.

\subsection{1. ${ }^{7} \mathrm{Li}(\mathrm{d}, \gamma){ }^{9} \mathrm{Be}$ affecting ${ }^{9} \mathrm{Be}$}

The total reaction rate consists of two contributions, namely a resonance and a direct part. The direct contribution is obtained by a numerical integration from the experimentally known $S$-factor (Schmid et al. 1993). The corresponding upper 
and lower limits are estimated by multiplying the S-factor by a factor 10 and 0.1 , respectively. The resonance contribution is estimated on the basis of Eqs. (11) and (14) in the NACRE evaluation (Angulo et al. 1999) where the resonance parameters and their uncertainties for the compound system ${ }^{9} \mathrm{Be}$ are taken from the RIPL-3 database (Capote et al. 2009). The final rate with the estimated uncertainties are shown and compared with TALYS predictions in Fig. 16.

\subsection{2. ${ }^{7} \mathrm{Li}(\mathrm{d}, \mathrm{n}) 2{ }^{4} \mathrm{He}$ affecting $\mathrm{CNO}$}

Both the resonant and direct mechanisms contribute to the total reaction rate. The resonance part is calculated by Eqs. (11) and (14) of Angulo et al. (1999), where the lowest 4 resonances in the ${ }^{7} \operatorname{Li}(\mathrm{d}, \mathrm{n}) 2 \alpha$ reaction centre-of-mass system are considered, the corresponding resonant parameters being taken from RIPL-3 Capote et al. (2009). For the direct part, the contribution is obtained by a numerical integration with a constant S-factor of $150 \mathrm{MeV}$ b is considered for the upper limit Hofstee et al. (2001) and of $5.4 \mathrm{MeV}$ b for the lower limit Sabourov et al. (2006). The recommended rate is obtained by the geometrical means of the lower and upper limits of the total rate. The final rate with the estimated uncertainties are shown and compared with the TALYS and Boyd et al. (1993) rates in Fig. 18.

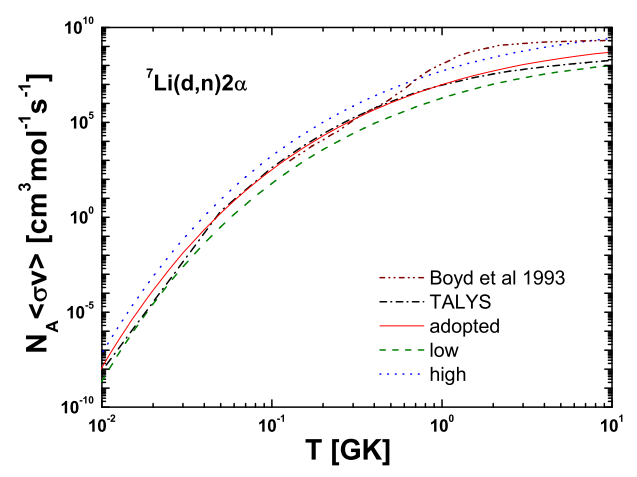

Fig. 18.- (Color online) Estimated rates for ${ }^{7} \mathrm{Li}(\mathrm{d}, \mathrm{n}) 2^{4} \mathrm{He}$ and comparison with TALYS and Boyd et al. (1993) rates.

\subsection{3. ${ }^{7} \mathrm{Li}(t, n)^{9} \mathrm{Be}$ affecting $\mathrm{CNO}$ and ${ }^{9} \mathrm{Be}$}

To estimate the ${ }^{7} \mathrm{Li}(\mathrm{t}, \mathrm{n}){ }^{9} \mathrm{~B}$ rate, experimental data from Brune et al. (1991), as well as theoretical calculations from Yamamoto et al. (1993) are considered. More precisely, the lower limit of the total reaction rate is obtained from the theoretical analysis of Yamamoto et al. (1993) based on the experimental determination of the ${ }^{7} \mathrm{Li}\left(\mathrm{t}, n_{0}\right){ }^{9} \mathrm{~B}$ cross section by Brune et al. (1991). The upper limit is assumed to be a factor of 25 larger than the lower limit. This factor corresponds to the ratio between the ${ }^{7} \operatorname{Li}\left(\mathrm{t}, n_{\text {tot }}\right){ }^{9} \mathrm{~B}$ and ${ }^{7} \mathrm{Li}\left(\mathrm{t}, n_{0}\right){ }^{9} \mathrm{~B}$ cross sections determined experimentally by Brune et al. (1991) in the 0.20 to $1.4 \mathrm{MeV}$ energy region. The final rates are shown in Fig.16.

\subsection{4. ${ }^{8} \operatorname{Li}(\alpha, n)^{11} B$ affecting $C N O$}

For this reaction, various experimental informations exist and may constrain the determination of the reaction rate. In particular, the ${ }^{8} \mathrm{Li}\left(\alpha, n_{\text {tot }}\right)^{11} \mathrm{~B}$ measurements of La Cognata et al. (2008, 2009) above typically $0.6 \mathrm{MeV}$ are used to estimate the upper limit of the cross section, considering a constant $S$-factor at energies below $0.6 \mathrm{MeV}$. This rate is also used as the recommended rate.

As far as the lower limit is concerned, experimental constraints are taken from the measurements of Ishivama et al. (2006) which are significantly lower than those obtained by La Cognata et al. (2008, 2009). At energies below $0.75 \mathrm{MeV}$, down to $0.4 \mathrm{MeV}$, the ground-state experimental cross section ${ }^{8} \mathrm{Li}\left(\alpha, n_{0}\right){ }^{11} \mathrm{~B}$ (Paradellis et al. 1990; Ishivama et al. 2006) provide a lower limit of the cross section, while at energies below $0.4 \mathrm{MeV}$, a constant $S$-factor is assumed and extrapolated from the Paradellis et al. (1990) and Ishiyama et al. (2006) data. The final rates are shown in Fig. 19] and compared with Gu et al. (1995) and Mizoi et al. (2000).

\subsection{5. ${ }^{7} \mathrm{Be}(t, p){ }^{9} \mathrm{Be}$ affecting ${ }^{9} \mathrm{Be}$}

The total rate also consists of a resonant plus a direct contribution. Since no experimental data is available, information on the direct contribution is taken from the mirror ${ }^{7} \mathrm{Li}\left({ }^{3} \mathrm{He}, \mathrm{p}\right){ }^{9} \mathrm{Be}$ reaction as given by Yamamoto et al. (1993). The upper and lower limits are estimated by multiplying the corresponding S-factor by factors of 10 and 0.1 , respectively. The same procedure as used for 


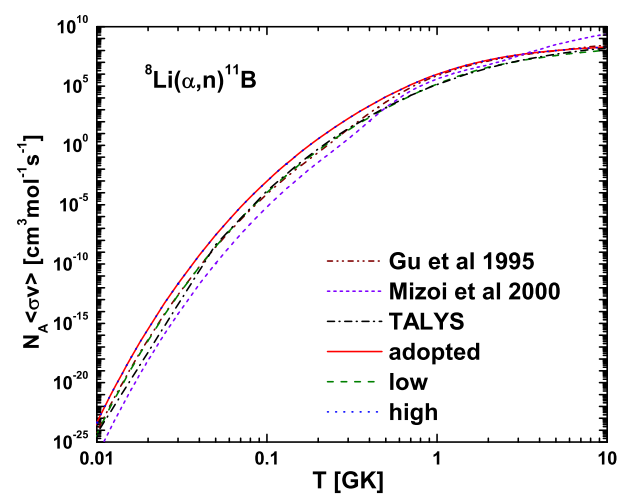

Fig. 19.- (Color online) Adopted rates for ${ }^{8} \mathrm{Li}(\alpha, n)^{11} \mathrm{~B}$ and comparison with TALYS , Gu et al. (1995) and Mizoi et al. (2000).

${ }^{7} \mathrm{Li}(\mathrm{d}, \gamma){ }^{9} \mathrm{Be}$ (see above) is followed to determine the resonance component, the resonant parameters of the compound nuclei ${ }^{10} \mathrm{~B}$ as well as their uncertainties being taken from the RIPL-3 library (Capote et al. 2009). The final rates are shown in Fig. 16 .

\subsection{6. ${ }^{11} B(n, \gamma)^{12} C$ affecting $C N O$}

A recent experimental determination of the ${ }^{11} \mathrm{~B}(\mathrm{n}, \gamma){ }^{12} \mathrm{C}$ cross section was obtained by Lee et al. (2010), including upper and lower limits (see Fig. 17). Those are considered in the present study.

\subsection{7. ${ }^{11} B(d, n)^{12} C$ affecting $C N O$}

For energies $E_{c m}$ ranging between 0.1 and 5 $\mathrm{MeV}$, five sets of experimental data are available (Ames et al. 1957; Siemssen et al. 1965; Class et al. 1965; Hofstee et al. 2001; Parpottas et al. 2006). A theoretical Distorted Wave Born Approximation (DWBA) analysis and extrapolation is performed to estimate the S-factors below $E_{c . m}=0.1 \mathrm{MeV}$, as detailed in Xu et al. (2010, 2011). The DWBA results (along with a $20 \%$ uncertainty) are compared with experimental data in Fig. 20. The final rates are computed by a numerical integration taking into account the DWBA estimates below $E_{c . m .}=0.5 \mathrm{MeV}$ and the experimental data with their error bars above (Fig. 20).

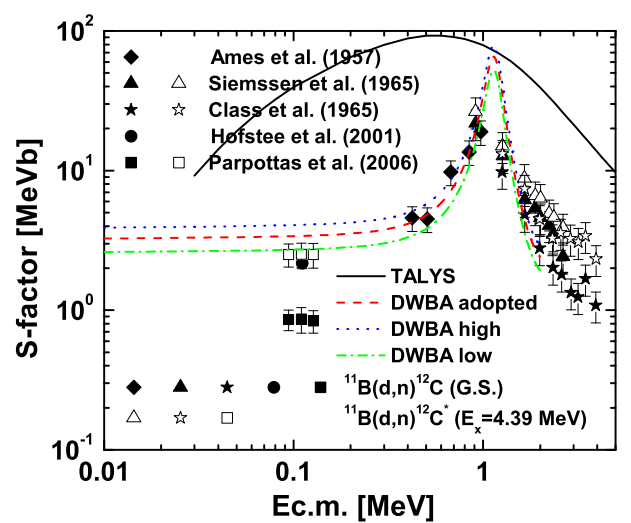

Fig. 20.- (Color online ${ }^{11} \mathrm{~B}(\mathrm{~d}, \mathrm{n}){ }^{12} \mathrm{C}$ experimental and estimated S-factor. The solid line corresponds to the TALYS prediction.

\subsection{8. ${ }^{11} B(d, p)^{12} B$ affecting $C N O$}

Three sets of experimental data have been reported (Kavanagh \& Barnes 1958; Guzhovskij et al. 1985; Yan et al. 1997) for energies $E_{c m}$ ranging from $0.1 \mathrm{MeV}$ to about $10 \mathrm{MeV}$. To extrapolate the S-factors below $E_{c . m .}=0.1 \mathrm{MeV}$, a DWBA evaluation is performed, as described above. The adopted results with an artificial $20 \%$ uncertainties are shown in Fig. 21 along with the available experimental data. The theoretical DWBA results below $E_{c m}=0.4 \mathrm{MeV}$ as well as the experimental data with the error bars above $E_{c m}=0.4 \mathrm{MeV}$ are used to estimate the final rates (see Fig. 21).

\subsection{9. ${ }^{11} C(n, \alpha) 2 \alpha$ affecting ${ }^{11} B$}

The total reaction rate consists of two contributions, namely a resonance and a direct component. Concerning the resonant contribution, the calculation is performed on the basis of Eqs. (11) and (14) of the NACRE compilation (Angulo et al. 1999), where the resonance parameters and their uncertainties in the compound nucleus ${ }^{12} \mathrm{C}$ are taken from the RIPL-3 library (Capote et al. 2009). The direct component (and the corresponding uncertainties) is evaluated based on the thermal cross sections (Rauscher et al. 1994). Thanks to the similar properties between the direct ${ }^{11} \mathrm{C}+\mathrm{n}$ and ${ }^{11} \mathrm{~B}+\mathrm{p}$ reaction channels, the thermal cross section is obtained separately by performing DWBA calculations for the ${ }^{11} \mathrm{C}(\mathrm{n}, \alpha) 2 \alpha$ reaction where the 


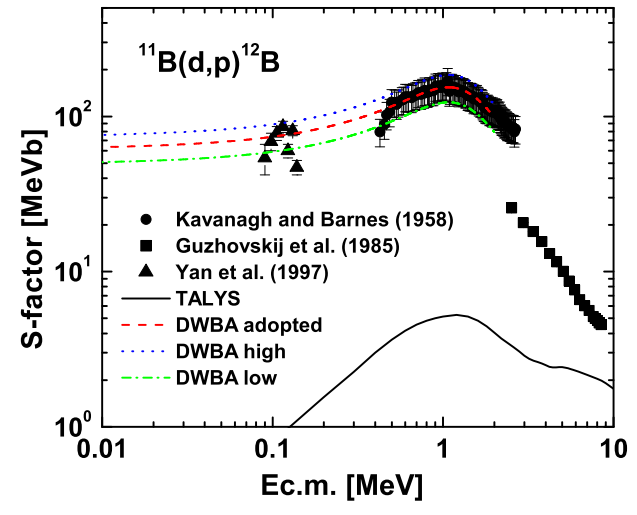

Fig. 21.- (Color online) ${ }^{11} \mathrm{~B}(\mathrm{~d}, \mathrm{p}){ }^{12} \mathrm{~B}$ experimental and estimated S-factor. The solid line corresponds to the TALYS prediction.

Woods-Saxon potential is constrained by experimental data on the mirror reaction ${ }^{11} \mathrm{~B}(\mathrm{p}, \alpha) 2 \alpha$, as described in the NACRE 2 compilation (Xu et al. 2011).

\subsubsection{0. ${ }^{11} C(d, p)^{12} C$ affecting $C N O$}

The total reaction rate consists of the two resonance and direct contributions. As done for previous reactions, for the resonance contribution, the parameters and their uncertainties in the compound nucleus ${ }^{13} \mathrm{~N}$ are taken from the RIPL-3 library (Capote et al. 2009). The direct component is obtained by a numerical integration with astrophysical factors $S(0)$ corresponding to 68.43 , 82.12 and $54.75 \mathrm{MeVb}$ for the recommended, upper and lower limits, respectively. These $S$-factors are taken from the direct component in the mirror reaction channel ${ }^{11} \mathrm{~B}(\mathrm{~d}, \mathrm{p}){ }^{12} \mathrm{~B}$, studied in Sect. 3.2.8.

Finally, the 6 charged-particle-induced reactions and 2 neutron-induced reactions that have been re-evaluated above are compared in Figs. 16] 17] with TALYS predictions. Some deviations are obtained essentially for the cases (e.g. $\left.{ }^{8} \operatorname{Li}(\alpha, n){ }^{11} \mathrm{~B}\right)$ where experimental data exist and have been used to constrain the reaction cross sections. For the other cases $\left(\right.$ e.g. $\left.{ }^{11} \mathrm{C}(\mathrm{d}, \mathrm{p}){ }^{12} \mathrm{C}\right)$, the new determination is also based on theoretical arguments and minor differences are found in fact.

\section{Results}

\subsection{Standard BBN and comparison with astrophysical constraints}

Table 2 displays the comparison between the spectroscopic observations and our BBN calculated ${ }^{4} \mathrm{He}, \mathrm{D},{ }^{3} \mathrm{He}$ and ${ }^{7} \mathrm{Li}$ abundances with i) our Monte-Carlo code including the 13 main nuclear reactions (Coc \& Vangioni 2010) and our new code with ii) the $13+2$ (for ${ }^{6} \mathrm{Li}$ ) nuclear reactions network, iii) the present extended (424) nuclear reaction network. The small difference between central Monte-Carlo values (CV10 column) and the new 15 reaction network results can be explained by the former use of the $\Omega_{\mathrm{b}} \cdot h^{2}=0.023$ value from Spergel et al. (2007) rather than the new WMAP 7 -years result $\Omega_{\mathrm{b}} \cdot h^{2}=0.02249$ Komatsu et al. 2011). The small difference $(\sim 1-2 \%)$ between the 13 and 424 reactions network can be traced back to the contribution of the additional reactions in the $\mathrm{A}<8$ domain. Nevertheless, Table 1 (where the threshold is at a $20 \%$ abundance variation) shows that none of these reactions can alleviate the lithium problem. We hence confirm the robustness of the standard BBN results and refer to a forthcoming paper the discussion of the ${ }^{4} \mathrm{He}, \mathrm{D}$, ${ }^{3} \mathrm{He}$ and ${ }^{7} \mathrm{Li}$ aspects. This is also true for the ${ }^{6} \mathrm{Li}$ abundance compared to Hammache et al. (2010). In particular, no new effective neutron source has been found that may destroy ${ }^{7} \mathrm{Be}$ and reduce the lithium problem.

The concordance with the spectroscopic observations is in perfect agreement for deuterium. Considering the large uncertainty associated with ${ }^{4} \mathrm{He}$ observations, the agreement is also fair. The calculated ${ }^{3} \mathrm{He}$ value is close to its galactic value showing that its abundance has not changed significantly during galactic chemical evolution. On the contrary, as well-known the ${ }^{7} \mathrm{Li}(\mathrm{CMB}+\mathrm{BBN})$ calculated abundance is significantly higher than the spectroscopic observations by a factor of about 3.5. Indeed the extended network cannot bring a sufficient neutron source to modify the primordial value. The origin of this discrepancy between $\mathrm{CMB}+\mathrm{BBN}$ and spectroscopic observations remains an open question. As mentioned before, Chakraborty et al. (2011) have proposed an efficient ${ }^{7} \mathrm{Be}$ destruction as a possible solution to the lithium problem; this destruction requires new resonances, in particular associated with the ${ }^{10} \mathrm{C}$ 
compound nucleus. In our sensitivity study, we have not seen such an effect that would require a rate enhancement by resonances larger than the three orders of magnitude considered here.

The main nuclear path to CNO (see also Iocco et al. 2007) proceeds from the ${ }^{7} \mathrm{Li}(\alpha, \gamma){ }^{11} \mathrm{~B}$ followed by ${ }^{11} \mathrm{~B}(\mathrm{p}, \gamma){ }^{12} \mathrm{C},{ }^{11} \mathrm{~B}(\mathrm{~d}, \mathrm{n}){ }^{12} \mathrm{C},{ }^{11} \mathrm{~B}(\mathrm{~d}, \mathrm{p}){ }^{12} \mathrm{~B}$ and ${ }^{11} \mathrm{~B}(\mathrm{n}, \gamma){ }^{12} \mathrm{~B}$ reactions. An other nucleosynthesis paths start with ${ }^{7} \operatorname{Li}(\mathrm{n}, \gamma){ }^{8} \operatorname{Li}(\alpha, \mathrm{n}){ }^{11} \mathrm{~B}$. The nuclear flow starting by ${ }^{8} \mathrm{~B}(\alpha, \gamma)^{12} \mathrm{~N}$ is negligible because it is well known (Hernanz et al. 1996) that ${ }^{8} \mathrm{~B}$ production by ${ }^{7} \mathrm{Be}(\mathrm{p}, \gamma)$ is hindered at high temperatures by photodisintegration, so that its formation is delayed until lower temperatures are reached (see Fig. 12). ${ }^{9} \mathrm{Be}$ is produced by ${ }^{7} \mathrm{Li}(\mathrm{t}, \mathrm{n}){ }^{9} \mathrm{Be}$ and ${ }^{7} \mathrm{Be}(\mathrm{t}, \mathrm{p}){ }^{9} \mathrm{Be}$ while final ${ }^{11} \mathrm{~B}$ is produced by the late decay of ${ }^{11} \mathrm{C}$ (see Fig. 12).

Table 3 compares the BeB and CNO primordial abundances calculated with our extended nuclear network in its initial version with those obtained after its improvement described in Section 3.2 and with the results of Iocco et al. (2007). As one can see, the final CNO abundances are very close, i.e $\mathrm{CNO} / \mathrm{H} \approx 5 \times 10^{-16}$ though some differences can be found in the isotopic composition. This is particularly visible for ${ }^{14} \mathrm{C}$ which is efficiently destroyed in our calculations (see Fig. 13) by the ${ }^{14} \mathrm{C}(\mathrm{p}, \gamma){ }^{15} \mathrm{~N}$ and ${ }^{14} \mathrm{C}(\mathrm{d}, \mathrm{n}){ }^{15} \mathrm{~N}$ reactions. ${ }^{14} \mathrm{C} / \mathrm{H}$ is found to be a factor $10^{4}$ more abundant than predicted by Iocco et al. (2007). The reason for this discrepancy is unknown as the Iocco et al. (2007) network is not given explicitly. For the sake of comparison, we also performed a nucleosynthesis calculation using Thomas et al. (1993, 1994) rates instead of the TALYS rates whenever available and found a ${ }^{10} \mathrm{~B} / \mathrm{H}$ abundance higher by a factor of $\sim 10^{4}$ and a CNO abundance higher by a factor of $\sim 10^{2}$. We will not discuss these results as they are due to quite unrealistic ${ }^{10} \mathrm{~B}(\alpha, \gamma){ }^{14} \mathrm{~N}$, ${ }^{10} \mathrm{Be}(\alpha, \gamma){ }^{14} \mathrm{C}$ and ${ }^{8} \mathrm{~B}(\alpha, \gamma){ }^{12} \mathrm{~N}$ rates (see Fig. 10).

\section{Conclusion}

We have used an extensive network of more than 400 nuclear reactions whose thermonuclear reaction rates are adopted from evaluations based on experimental data or were calculated using the TALYS code. It enabled us to calculate the Standard Big-Bang Nucleosynthesis production of ${ }^{6} \mathrm{Li}$, ${ }^{9} \mathrm{Be},{ }^{10} \mathrm{~B},{ }^{11} \mathrm{~B}$ and $\mathrm{CNO}$ isotopes more reliably.
We performed a sensitivity study by varying uncertain reaction rates by factors of up to 1000 and down to 0.001 . In that way, a few reactions that could affect the $\mathrm{A}>7$ isotope yields were identified and their rate (re-)evaluated using available experimental data and/or theoretical or phenomenological input. On the basis of these new evaluations the ${ }^{9} \mathrm{Be},{ }^{10} \mathrm{~B},{ }^{11} \mathrm{~B}$ and $\mathrm{CNO}$ isotope production was found to be close to the initial results and in global agreement with previous calculations (Iocco et al. 2007).

For most of the few reactions which were identified to have an impact on Standard Big-Bang Nucleosynthesis, we were able to collect sufficient experimental data to derive new reaction rates with associated uncertainties much reduced with respect to our initial three orders of magnitude variation. (An exception is ${ }^{7} \mathrm{Be}(\mathrm{t}, \mathrm{p}){ }^{9} \mathrm{Be}$ but affecting the ${ }^{9} \mathrm{Be}$ production only.) In some cases these new rates differ from the previous ones by large factors but changes compensating each other (e.g. ${ }^{11} \mathrm{~B}(\mathrm{~d}, \mathrm{n}){ }^{12} \mathrm{C}$ and $\left.{ }^{11} \mathrm{~B}(\mathrm{~d}, \mathrm{p}){ }^{12} \mathrm{~B}\right)$ allow us to confirm the CNO Standard Big-Bang Nucleosynthesis production of about $0.7 \times 10^{-14}$ in mass fraction (i.e $\mathrm{CNO} / \mathrm{H} \approx 0.7 \times 10^{-15}$ ). Based on the rate uncertainties (a factor of $\lesssim 10$ at BBN temperatures) obtained in Section 3.2 and combining (see Longland et al. 2010) the corresponding uncertainty factors from Table 1 we can estimate the uncertainty on CNO production to be of a factor of $\lesssim 4$. This is too small to have presently an impact on Population III stellar evolution. It is nevertheless a reference value for comparison with nonStandard Big-Bang Nucleosynthesis CNO production e.g. in the context of varying constants.

Finally, our extension of the network does not help alleviate the discrepancy between the calculated and observed ${ }^{7} \mathrm{Li}$ abundances. No new latetime neutron source was found that could destroy ${ }^{7} \mathrm{Be}$ before it decays to ${ }^{7} \mathrm{Li}$.

Nevertheless we pointed out the unexpected high sensitivity of the CNO abundance with respect to the ${ }^{7} \operatorname{Li}(\mathrm{d}, \mathrm{n}) 2{ }^{4} \mathrm{He}$ reaction rate. A similar situation was found between the ${ }^{7} \mathrm{Li}$ abundance and the ${ }^{1} \mathrm{H}(\mathrm{n}, \gamma)^{2} \mathrm{H}$ reaction rate. This emphasizes again the complex nature of nucleosynthesis and a posteriori justifies such a sensitivity study: the impact of a given reaction being not always predictable, even in the simple case (i.e. homogeneity, no mixing, nor convection,...) of BBN. As a conse- 
quence, even though very unlikely, the search for a nuclear solution to the lithium problem remains justified.

Acknowledgements. We apologize in advance for unintentionally omitting references to evaluated reaction rates unknown to us. S.G. and X.Y. acknowledge the financial support of the "Actions de recherche concertées (ARC)" from the "Communauté française de Belgique". S.G is F.N.R.S. research associate. 


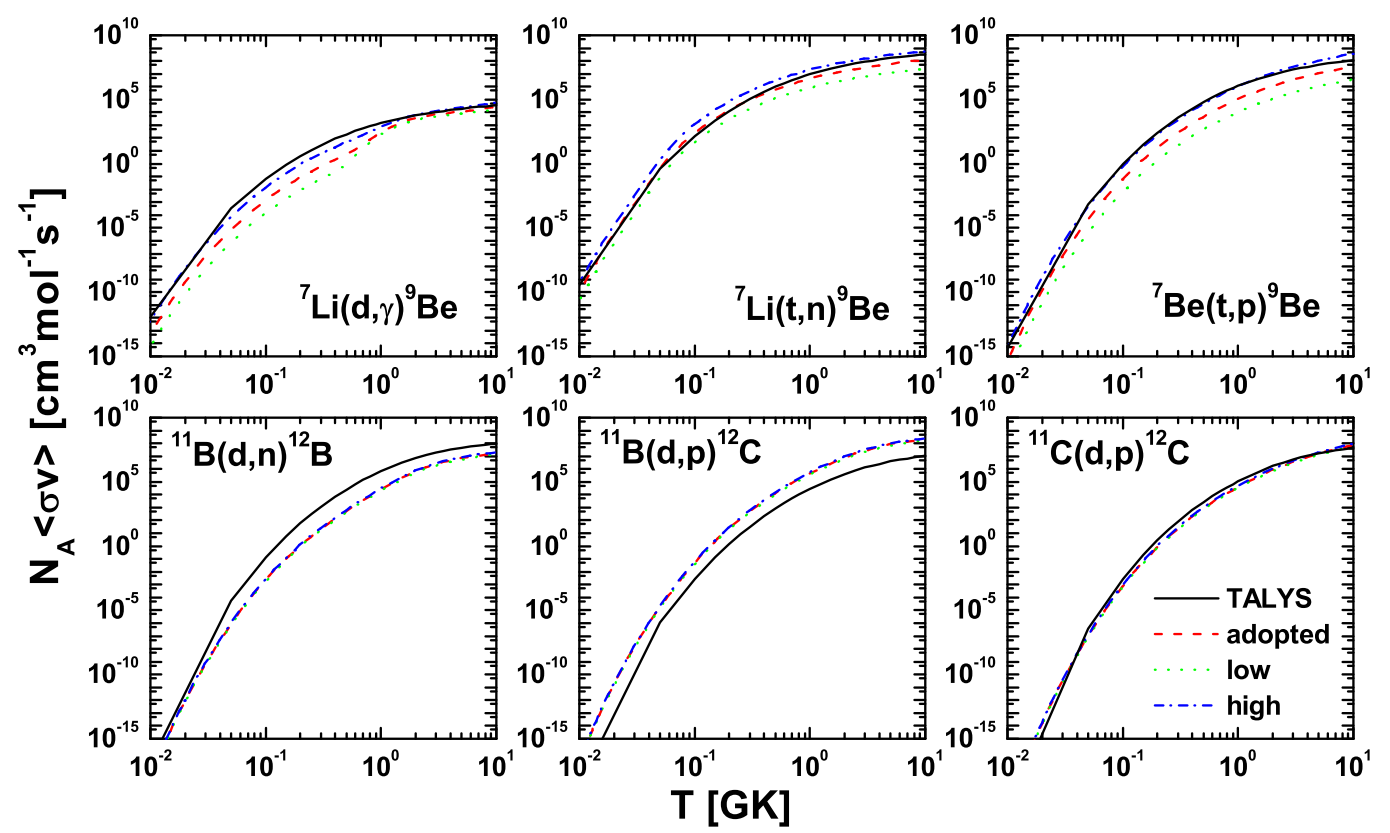

Fig. 16. - (Color online) Comparison of the newly determined rates (dashed line) and their estimated uncertainties (dash-dot and dotted lines) with TALYS predictions (solid line) for the six reactions ${ }^{7} \mathrm{Li}(\mathrm{d}, \gamma)^{9} \mathrm{Be}$, ${ }^{7} \mathrm{Li}(\mathrm{t}, \mathrm{n}){ }^{9} \mathrm{~B},{ }^{7} \mathrm{Be}(\mathrm{t}, \mathrm{p}){ }^{9} \mathrm{Be},{ }^{11} \mathrm{~B}(\mathrm{~d}, \mathrm{n}){ }^{12} \mathrm{C},{ }^{11} \mathrm{~B}(\mathrm{~d}, \mathrm{p}){ }^{12} \mathrm{~B},{ }^{11} \mathrm{C}(\mathrm{d}, \mathrm{p}){ }^{12} \mathrm{C}$.

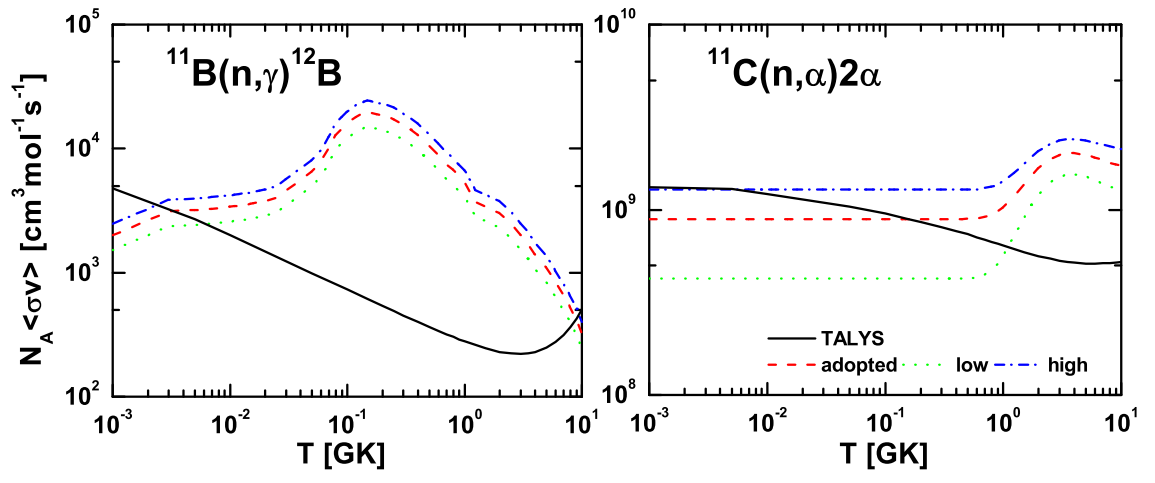

Fig. 17.- (Color online) Same as Fig. 16 for ${ }^{11} \mathrm{~B}(\mathrm{n}, \gamma){ }^{12} \mathrm{C}$ and ${ }^{11} \mathrm{C}(\mathrm{n}, \alpha) 2 \alpha$. 


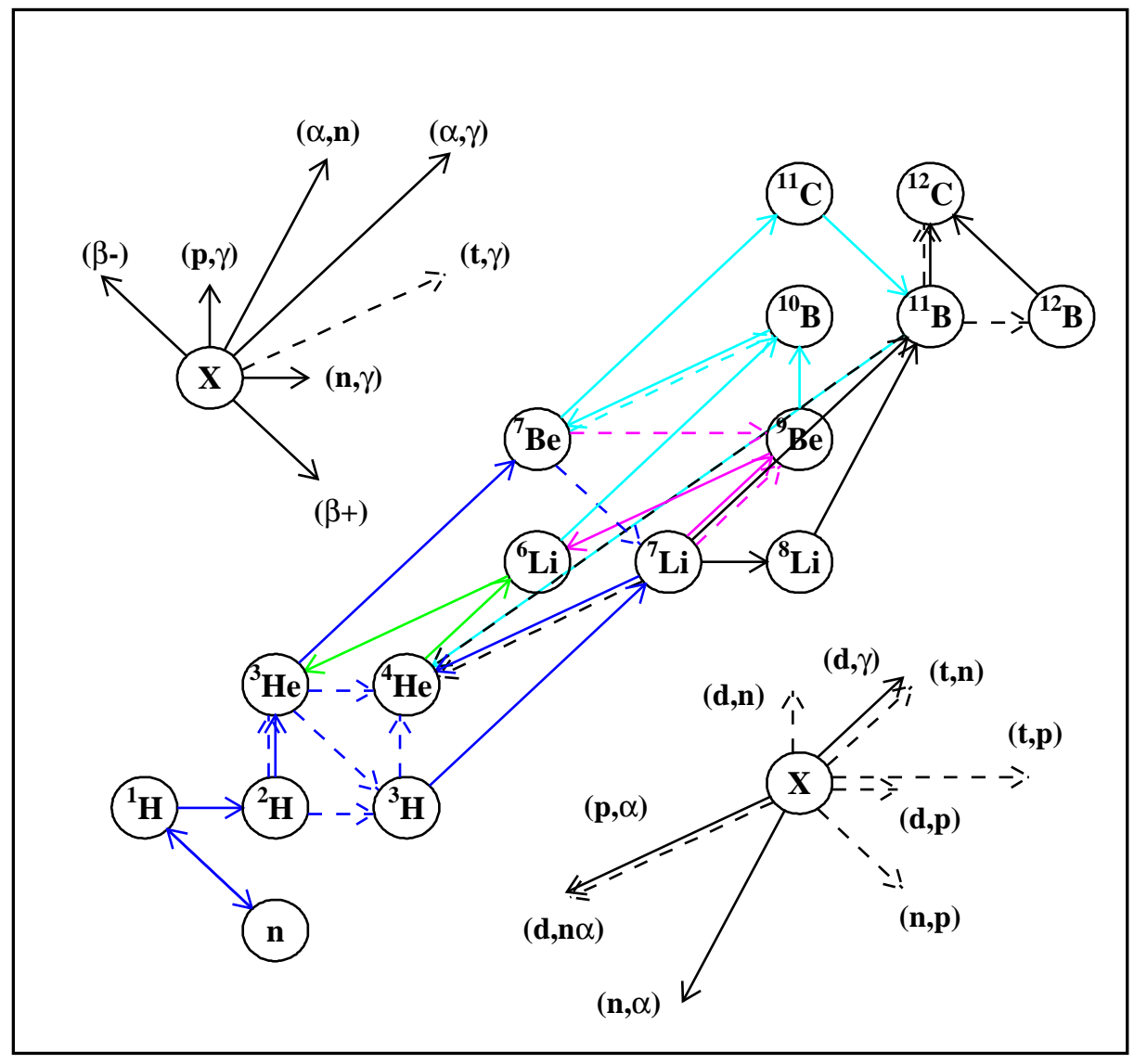

Fig. 22. - (Color online) Reduced network displaying the important reactions for ${ }^{4} \mathrm{He}, \mathrm{D},{ }^{3} \mathrm{He}$ and ${ }^{7} \mathrm{Li}$ (blue), ${ }^{6} \mathrm{Li}$ (green), ${ }^{9} \mathrm{Be}$ (pink), ${ }^{10,11} \mathrm{~B}$ (cyan) and CNO (black) production. Note that CNO production is via ${ }^{11} \mathrm{~B}$ but follows a different path than primordial ${ }^{11} \mathrm{~B}$ formation through the late time ${ }^{11} \mathrm{C}$ decay. 
Table 2: Primordial abundances of $\mathrm{H}, \mathrm{He}$ and Li isotopes at WMAP7 baryonic density.

\begin{tabular}{ccccc}
\hline & CV10 & This work & This work & Observations \\
Nb. reactions & $13(+2)$ & 15 & 424 & \\
\hline$Y_{p}$ & $0.2476 \pm 0.0004$ & 0.2475 & 0.2476 & $0.2561 \pm 0.0108$ \\
$\mathrm{D} / \mathrm{H}\left(\times 10^{-5}\right)$ & $2.68 \pm 0.15$ & 2.64 & 2.59 & $2.82 \pm 0.2$ \\
${ }^{3} \mathrm{He} / \mathrm{H}\left(\times 10^{-5}\right)$ & $1.05 \pm 0.04$ & 1.05 & 1.04 & $1.1 \pm 0.2$ \\
${ }^{7} \mathrm{Li} / \mathrm{H}\left(\times 10^{-10}\right)$ & $5.14 \pm 0.50$ & 5.20 & 5.24 & $1.58 \pm 0.31$ \\
${ }^{6} \mathrm{Li} / \mathrm{H}\left(\times 10^{-14}\right)$ & $1.3^{\dagger}$ & 1.32 & 1.23 & $\sim 1000(?)$ \\
\hline
\end{tabular}

CV10: Coc \& Vangioni (2010) using $\Omega_{\mathrm{b}} \cdot h^{2}$ from Spergel et al. (2007). This work uses the new Komatsu et al. (2011) value.

Hammache et al. (2010)

Table 3: Be to CNO primordial abundances by number at WMAP7 baryonic density.

\begin{tabular}{|c|c|c|c|}
\hline & IMMPS07 & $\begin{array}{c}\text { This work } \\
\text { (Initial*) }\end{array}$ & $\begin{array}{l}\text { This work } \\
\text { (Improved }^{*} \text { ) }\end{array}$ \\
\hline${ }^{9} \mathrm{Be} / \mathrm{H}\left(\times 10^{-19}\right)$ & 2.5 & 2.24 & 9.60 \\
\hline${ }^{10} \mathrm{~B} / \mathrm{H}\left(\times 10^{-21}\right)$ & & 2.78 & 3.00 \\
\hline${ }^{11} \mathrm{~B} / \mathrm{H}\left(\times 10^{-16}\right)$ & 3.9 & 5.86 & 3.05 \\
\hline${ }^{12} \mathrm{C} / \mathrm{H}\left(\times 10^{-16}\right)$ & 4.6 & 3.56 & 5.34 \\
\hline${ }^{13} \mathrm{C} / \mathrm{H}\left(\times 10^{-16}\right)$ & 0.90 & 0.87 & 1.41 \\
\hline${ }^{14} \mathrm{C} / \mathrm{H}\left(\times 10^{-21}\right)$ & 13000. & 0.96 & 1.62 \\
\hline${ }^{14} \mathrm{~N} / \mathrm{H}\left(\times 10^{-17}\right)$ & 3.7 & 3.98 & 6.76 \\
\hline${ }^{15} \mathrm{~N} / \mathrm{H}\left(\times 10^{-20}\right)$ & & 1.32 & 2.25 \\
\hline${ }^{16} \mathrm{O} / \mathrm{H}\left(\times 10^{-20}\right)$ & 2.7 & 5.18 & 9.13 \\
\hline $\mathrm{CNO} / \mathrm{H}\left(\times 10^{-16}\right)$ & 6.00 & 4.83 & 7.43 \\
\hline
\end{tabular}

IMMPS07: Iocco et al. $(2007)$ C3 code.

*'Initial' corresponds to original network before re-evaluations of selected reaction rates 'improved' (see text). 
A. Network 
TABle 4

Network

\begin{tabular}{|c|c|c|c|c|c|}
\hline Reaction & Ref. & $\mathrm{Q}(\mathrm{MeV})$ & Reaction & Ref. & $\mathrm{Q}(\mathrm{MeV})$ \\
\hline${ }^{1} \mathrm{n}(\mathrm{p}, \gamma)^{2} \mathrm{H}$ & And06 & 2.2246 & ${ }^{1} \mathrm{H}(\mathrm{n}, \mathrm{p})^{2} \mathrm{H}$ & CF88 & 2.2246 \\
\hline${ }^{2} \mathrm{H}(\mathrm{n}, \gamma){ }^{3} \mathrm{H}$ & Nag06 & 6.2572 & ${ }^{2} \mathrm{H}(\mathrm{p}, \gamma){ }^{3} \mathrm{He}$ & DAACV04 & 5.4935 \\
\hline${ }^{2} \mathrm{H}(\mathrm{d}, \mathrm{n}){ }^{3} \mathrm{He}$ & DAACV04 & 3.2689 & ${ }^{2} \mathrm{H}(\mathrm{d}, \mathrm{p}){ }^{3} \mathrm{H}$ & DAACV04 & 4.0327 \\
\hline${ }^{2} \mathrm{H}(\alpha, \gamma){ }^{6} \mathrm{Li}$ & Ham10 & 1.4738 & ${ }^{2} \mathrm{H}(\mathrm{d}, \gamma){ }^{4} \mathrm{He}$ & NACREII & 23.8465 \\
\hline${ }^{3} \mathrm{H}(\mathrm{t}, 2 \mathrm{n})^{4} \mathrm{He}$ & CF88 & 11.3321 & ${ }^{3} \mathrm{H}(\alpha, n)^{6} \mathrm{Li}$ & CF88 & -4.7834 \\
\hline${ }^{3} \mathrm{H}(\mathrm{p}, \gamma){ }^{4} \mathrm{He}$ & Ser04 & 19.8139 & ${ }^{3} \mathrm{H}(\mathrm{d}, \mathrm{n}){ }^{4} \mathrm{He}$ & DAACV04 & 17.5893 \\
\hline${ }^{3} \mathrm{H}(\alpha, \gamma){ }^{7} \mathrm{Li}$ & DAACV04 & 2.4666 & ${ }^{3} \mathrm{He}(\mathrm{n}, \gamma){ }^{4} \mathrm{He}$ & ?Wag69 & 20.5776 \\
\hline${ }^{3} \mathrm{He}(\mathrm{t}, \mathrm{d}){ }^{4} \mathrm{He}$ & CF88 & 14.3204 & ${ }^{3} \mathrm{He}(\mathrm{t}, \mathrm{np})^{4} \mathrm{He}$ & CF88 & 12.0958 \\
\hline${ }^{3} \mathrm{He}(\mathrm{t}, \gamma){ }^{6} \mathrm{Li}$ & FK90 & 15.7942 & ${ }^{3} \mathrm{He}(\mathrm{n}, \mathrm{p})^{3} \mathrm{H}$ & DAACV04 & 0.7638 \\
\hline${ }^{3} \mathrm{He}(\mathrm{d}, \mathrm{p})^{4} \mathrm{He}$ & DAACV04 & 18.3531 & ${ }^{3} \mathrm{He}(\alpha, \gamma){ }^{7} \mathrm{Be}$ & Cyb08 & 1.5861 \\
\hline${ }^{3} \mathrm{He}\left({ }^{3} \mathrm{He}, 2 \mathrm{p}\right){ }^{4} \mathrm{He}$ & NACREII & 12.8596 & ${ }^{4} \mathrm{He}(\alpha \mathrm{n}, \gamma){ }^{9} \mathrm{Be}$ & NACRE & 1.5735 \\
\hline${ }^{4} \mathrm{He}(\mathrm{np}, \gamma){ }^{6} \mathrm{Li}$ & CF88! & 3.6984 & ${ }^{4} \mathrm{He}\left(\alpha \alpha, \gamma^{12} \mathrm{C}\right.$ & NACRE & 7.2747 \\
\hline${ }^{4} \mathrm{He}(2 \mathrm{n}, \gamma){ }^{6} \mathrm{He}$ & Efr96 & 0.9724 & ${ }^{6} \mathrm{Li}(\mathrm{n}, \gamma){ }^{7} \mathrm{Li}$ & MF89 & 7.2500 \\
\hline${ }^{6} \mathrm{Li}(\mathrm{d}, \mathrm{p}){ }^{7} \mathrm{Li}$ & MF89 & 5.0254 & ${ }^{6} \mathrm{Li}(\mathrm{d}, \mathrm{n})^{7} \mathrm{Be}$ & MF89 & 3.3812 \\
\hline${ }^{6} \mathrm{Li}(\alpha, \gamma){ }^{10} \mathrm{~B}$ & CF88 & 4.4610 & ${ }^{6} \mathrm{Li}(\mathrm{p}, \gamma){ }^{7} \mathrm{Be}$ & NACREII & 5.6057 \\
\hline${ }^{6} \mathrm{Li}(\mathrm{p}, \alpha)^{3} \mathrm{He}$ & NACREII & 4.0196 & ${ }^{6} \mathrm{Li}\left({ }^{3} \mathrm{He}, \mathrm{p}\right){ }^{4} \mathrm{He}$ & TALYS & 16.8792 \\
\hline${ }^{6} \mathrm{Li}(\mathrm{t}, \gamma){ }^{9} \mathrm{Be}$ & TALYS & 17.6890 & ${ }^{6} \mathrm{Li}(\mathrm{t}, \mathrm{n}){ }^{4} \mathrm{He}$ & TALYS & 16.1155 \\
\hline${ }^{6} \mathrm{Li}(\mathrm{t}, \mathrm{p})^{8} \mathrm{Li}$ & TALYS & 0.8008 & ${ }^{6} \mathrm{Li}(\mathrm{t}, \mathrm{d})^{7} \mathrm{Li}$ & TALYS & 0.9927 \\
\hline${ }^{6} \mathrm{Li}\left({ }^{3} \mathrm{He}, \mathrm{d}\right){ }^{7} \mathrm{Be}$ & TALYS & 0.1123 & ${ }^{7} \mathbf{L i}(\mathbf{d}, \mathbf{n}) 2^{4} \mathbf{H e}$ & Boy93 & 15.1227 \\
\hline${ }^{7} \mathrm{Li}(\mathrm{t}, 2 \mathrm{n}){ }^{4} \mathrm{He}$ & CF88\& MF89 & 8.8655 & ${ }^{7} \mathrm{Li}\left({ }^{3} \mathrm{He}, \mathrm{np}\right){ }^{4} \mathrm{He}$ & CF88\& MF89 & 9.6292 \\
\hline${ }^{7} \mathbf{L i}(\mathbf{t}, \mathbf{n}){ }^{9} \mathbf{B e}$ & Bru91 & 10.4390 & ${ }^{7} \mathrm{Li}(\alpha, n){ }^{10} \mathrm{~B}$ & NACRE & -2.7890 \\
\hline${ }^{7} \mathrm{Li}(\mathrm{n}, \gamma)^{8} \mathrm{Li}$ & MF89Hei98 & 2.0326 & ${ }^{7} \mathrm{Li}(\mathrm{d}, \mathrm{p})^{8} \mathrm{Li}$ & MF89 & -0.1919 \\
\hline${ }^{7} \mathrm{Li}(\mathrm{p}, \alpha)^{4} \mathrm{He}$ & DAACV04 & 17.3473 & ${ }^{7} \mathrm{Li}(\mathrm{p}, \gamma){ }^{4} \mathrm{He}$ & NACREII & 17.3473 \\
\hline${ }^{7} \mathbf{L i}(\mathbf{d}, \gamma){ }^{9} \mathbf{B e}$ & TALYS & 16.6963 & ${ }^{7} \mathrm{Li}\left({ }^{3} \mathrm{He}, \gamma\right){ }^{10} \mathrm{~B}$ & TALYS & 17.7887 \\
\hline${ }^{7} \mathrm{Li}\left({ }^{3} \mathrm{He}, \alpha\right){ }^{6} \mathrm{Li}$ & TALYS & 13.3276 & ${ }^{7} \mathrm{Li}(\mathrm{t}, \gamma){ }^{10} \mathrm{Be}$ & TALYS & 17.2512 \\
\hline${ }^{7} \mathrm{Li}\left({ }^{3} \mathrm{He}, \mathrm{p}\right){ }^{9} \mathrm{Be}$ & TALYS & 11.2028 & ${ }^{7} \mathrm{Li}\left({ }^{3} \mathrm{He}, \mathrm{d}\right){ }^{4} \mathrm{He}$ & TALYS & 11.8538 \\
\hline${ }^{7} \operatorname{Li}(\alpha, \gamma){ }^{11} \mathrm{~B}$ & NACREII & 8.6652 & ${ }^{8} \mathrm{Li}(\mathrm{d}, \mathrm{p}){ }^{9} \mathrm{Li}$ & Bal95 & 1.8393 \\
\hline${ }^{8} \mathrm{Li}(\mathrm{d}, \mathrm{t})^{7} \mathrm{Li}$ & Has09 & 4.2246 & ${ }^{8} \mathrm{Li}(\mathrm{n}, \gamma){ }^{9} \mathrm{Li}$ & Rau94 & 4.0639 \\
\hline${ }^{8} \mathrm{Li}(\mathrm{p}, \mathrm{n})^{4} \mathrm{He}$ & Bec92 & 15.3147 & ${ }^{8} \mathrm{Li}(\mathrm{d}, \mathrm{n}){ }^{9} \mathrm{Be}$ & Bal95 & 14.6636 \\
\hline${ }^{8} \mathrm{Li}(\mathrm{p}, \gamma){ }^{9} \mathrm{Be}$ & TUNL\& Cam08 & 16.8882 & ${ }^{8} \mathrm{Li}(\alpha, \gamma){ }^{12} \mathrm{~B}$ & TALYS & 10.0029 \\
\hline${ }^{8} \mathbf{L i}(\alpha, \mathbf{n}){ }^{11} \mathbf{B}$ & Miz00 & 6.6325 & ${ }^{8} \mathrm{Li}(\mathrm{d}, \gamma){ }^{10} \mathrm{Be}$ & TALYS & 21.4759 \\
\hline${ }^{8} \mathrm{Li}\left({ }^{3} \mathrm{He}, \gamma\right){ }^{11} \mathrm{~B}$ & TALYS & 27.2102 & ${ }^{8} \mathrm{Li}\left({ }^{3} \mathrm{He}, \mathrm{n}\right){ }^{10} \mathrm{~B}$ & TALYS & 15.7560 \\
\hline${ }^{8} \mathrm{Li}\left({ }^{3} \mathrm{He}, \mathrm{p}\right){ }^{10} \mathrm{Be}$ & TALYS & 15.9824 & ${ }^{8} \mathrm{Li}\left({ }^{3} \mathrm{He}, \alpha\right){ }^{7} \mathrm{Li}$ & TALYS & 18.5450 \\
\hline${ }^{8} \mathrm{Li}(\mathrm{t}, \gamma){ }^{11} \mathrm{Be}$ & TALYS & 15.7226 & ${ }^{8} \mathrm{Li}(\mathrm{t}, \mathrm{n}){ }^{10} \mathrm{Be}$ & TALYS & 15.2186 \\
\hline${ }^{8} \mathrm{Li}\left({ }^{3} \mathrm{He}, \mathrm{d}\right){ }^{9} \mathrm{Be}$ & TALYS & 11.3947 & ${ }^{8} \mathrm{Li}\left({ }^{3} \mathrm{He}, \mathrm{t}\right){ }^{4} \mathrm{He}$ & TALYS & 16.0784 \\
\hline${ }^{9} \mathrm{Li}(\mathrm{p}, \mathrm{t})^{7} \mathrm{Li}$ & TALYS & 2.3853 & ${ }^{9} \mathrm{Li}(\mathrm{d}, \mathrm{n}){ }^{10} \mathrm{Be}$ & TALYS & 17.4120 \\
\hline${ }^{9} \mathrm{Li}(\mathrm{p}, \mathrm{n}){ }^{9} \mathrm{Be}$ & TALYS & 12.8244 & ${ }^{9} \operatorname{Li}(\alpha, n){ }^{12} \mathrm{~B}$ & TALYS & 5.9390 \\
\hline${ }^{9} \mathrm{Li}(\mathrm{p}, \gamma){ }^{10} \mathrm{Be}$ & TALYS & 19.6366 & ${ }^{9} \mathrm{Li}(\alpha, \gamma){ }^{13} \mathrm{~B}$ & TALYS & 10.8170 \\
\hline${ }^{9} \mathrm{Li}(\mathrm{d}, \gamma){ }^{11} \mathrm{Be}$ & TALYS & 17.9160 & ${ }^{9} \mathrm{Li}\left({ }^{3} \mathrm{He}, \gamma\right){ }^{12} \mathrm{~B}$ & TALYS & 26.5166 \\
\hline${ }^{9} \mathrm{Li}\left({ }^{3} \mathrm{He}, \mathrm{n}\right){ }^{11} \mathrm{~B}$ & TALYS & 23.1463 & ${ }^{9} \mathrm{Li}\left({ }^{3} \mathrm{He}, \mathrm{p}\right){ }^{11} \mathrm{Be}$ & TALYS & 12.4225 \\
\hline${ }^{9} \mathrm{Li}\left({ }^{3} \mathrm{He}, \alpha\right){ }^{8} \mathrm{Li}$ & TALYS & 16.5138 & ${ }^{9} \mathrm{Li}(\mathrm{t}, \gamma){ }^{12} \mathrm{Be}$ & TALYS & 14.8271 \\
\hline${ }^{9} \mathrm{Li}(\mathrm{t}, \mathrm{n}){ }^{11} \mathrm{Be}$ & TALYS & 11.6588 & ${ }^{9} \mathrm{Li}(\mathrm{d}, \mathrm{t})^{8} \mathrm{Li}$ & TALYS & 2.1934 \\
\hline${ }^{9} \mathrm{Li}\left({ }^{3} \mathrm{He}, \mathrm{d}\right){ }^{10} \mathrm{Be}$ & TALYS & 14.1431 & ${ }^{9} \mathrm{Li}\left({ }^{3} \mathrm{He}, \mathrm{t}\right){ }^{9} \mathrm{Be}$ & TALYS & 13.5881 \\
\hline
\end{tabular}


TABle 4-Continued

\begin{tabular}{|c|c|c|c|c|c|}
\hline${ }^{7} \mathrm{Be}(\mathrm{n}, \mathrm{p}){ }^{7} \mathrm{Li}$ & DAACV04 & 1.6442 & ${ }^{7} \mathrm{Be}(\mathrm{d}, \mathrm{p}){ }^{4} \mathrm{He}$ & CF88 & 16.7670 \\
\hline${ }^{7} \mathrm{Be}(\mathrm{t}, \mathrm{np}){ }^{4} \mathrm{He}$ & CF88\& MF89 & 10.5097 & ${ }^{7} \mathrm{Be}\left({ }^{3} \mathrm{He}, 2 \mathrm{p}\right){ }^{4} \mathrm{He}$ & CF88\& MF89 & 11.2735 \\
\hline${ }^{7} \mathrm{Be}\left({ }^{3} \mathrm{He}, \gamma\right){ }^{10} \mathrm{C}$ & TALYS & 15.0025 & ${ }^{7} \mathrm{Be}(\mathrm{n}, \gamma){ }^{4} \mathrm{He}$ & TALYS & 18.9915 \\
\hline${ }^{7} \mathrm{Be}(\mathrm{t}, \gamma){ }^{10} \mathrm{~B}$ & TALYS & 18.6691 & ${ }^{7} \mathrm{Be}(\mathrm{t}, \mathrm{p}){ }^{9} \mathrm{Be}$ & TALYS & 12.0833 \\
\hline${ }^{7} \mathrm{Be}(\mathrm{t}, \alpha){ }^{6} \mathrm{Li}$ & TALYS & 14.2081 & ${ }^{7} \mathrm{Be}(\mathrm{t}, \mathrm{d}){ }^{4} \mathrm{He}$ & TALYS & 12.7343 \\
\hline${ }^{7} \mathrm{Be}\left(\mathrm{t},{ }^{3} \mathrm{He}\right){ }^{7} \mathrm{Li}$ & TALYS & 0.8805 & ${ }^{7} \mathrm{Be}\left({ }^{3} \mathrm{He}, \mathrm{p}\right) 2 \alpha$ & TALYS & 11.2735 \\
\hline${ }^{7} \mathrm{Be}(\mathrm{p}, \gamma){ }^{8} \mathrm{~B}$ & NACREII & 0.1375 & ${ }^{7} \mathrm{Be}(\alpha, \gamma){ }^{11} \mathrm{C}$ & NACREII & 7.5446 \\
\hline${ }^{9} \mathrm{Be}(\mathrm{n}, \gamma){ }^{10} \mathrm{Be}$ & Rau94 & 6.8122 & ${ }^{9} \mathrm{Be}(\mathrm{p}, \mathrm{pn}){ }^{4} \mathrm{He}$ & NACRE & -1.5735 \\
\hline${ }^{9} \mathrm{Be}(\mathrm{t}, \mathrm{n}){ }^{11} \mathrm{~B}$ & TALYS & 9.5582 & ${ }^{9} \mathrm{Be}(\alpha, \gamma){ }^{13} \mathrm{C}$ & TALYS & 10.6475 \\
\hline${ }^{9} \mathrm{Be}(\mathrm{d}, \gamma){ }^{11} \mathrm{~B}$ & TALYS & 15.8154 & ${ }^{9} \mathrm{Be}(\mathrm{d}, \mathrm{n}){ }^{10} \mathrm{~B}$ & TALYS & 4.3613 \\
\hline${ }^{9} \mathrm{Be}(\mathrm{d}, \mathrm{p}){ }^{10} \mathrm{Be}$ & TALYS & 4.5877 & ${ }^{9} \mathrm{Be}(\mathrm{d}, \alpha){ }^{7} \mathrm{Li}$ & TALYS & 7.1503 \\
\hline${ }^{9} \mathrm{Be}\left({ }^{3} \mathrm{He}, \gamma\right){ }^{12} \mathrm{C}$ & TALYS & 26.2788 & ${ }^{9} \mathrm{Be}\left({ }^{3} \mathrm{He}, \mathrm{n}\right){ }^{11} \mathrm{C}$ & TALYS & 7.5572 \\
\hline${ }^{9} \mathrm{Be}\left({ }^{3} \mathrm{He}, \mathrm{p}\right){ }^{11} \mathrm{~B}$ & TALYS & 10.3219 & ${ }^{9} \mathrm{Be}\left({ }^{3} \mathrm{He}, \alpha\right){ }^{4} \mathrm{He}$ & TALYS & 19.0041 \\
\hline${ }^{9} \mathrm{Be}(\mathrm{t}, \gamma){ }^{12} \mathrm{~B}$ & TALYS & 12.9285 & ${ }^{9} \mathrm{Be}(\mathrm{t}, \alpha)^{8} \mathrm{Li}$ & TALYS & 2.9257 \\
\hline${ }^{9} \mathrm{Be}(\mathrm{d}, \mathrm{t}){ }^{4} \mathrm{He}$ & TALYS & 4.6837 & ${ }^{9} \mathrm{Be}(\mathrm{t}, \mathrm{d}){ }^{10} \mathrm{Be}$ & TALYS & 0.5550 \\
\hline${ }^{9} \mathrm{Be}\left({ }^{3} \mathrm{He}, \mathrm{d}\right){ }^{10} \mathrm{~B}$ & TALYS & 1.0924 & ${ }^{9} \mathrm{Be}(\mathrm{p}, \gamma){ }^{10} \mathrm{~B}$ & NACREII & 6.5859 \\
\hline${ }^{9} \mathrm{Be}(\mathrm{p}, \mathrm{d}){ }^{4} \mathrm{He}$ & NACREII & 0.6510 & ${ }^{9} \mathrm{Be}(\mathrm{p}, \alpha){ }^{6} \mathrm{Li}$ & NACREII & 2.1249 \\
\hline${ }^{9} \mathrm{Be}(\alpha, \mathrm{n}){ }^{12} \mathrm{C}$ & NACREII & 5.7012 & ${ }^{10} \mathrm{Be}(\mathrm{n}, \gamma){ }^{11} \mathrm{Be}$ & Rau94 & 0.5040 \\
\hline${ }^{10} \operatorname{Be}(\alpha, \gamma){ }^{14} \mathrm{C}$ & TALYS & 12.0117 & ${ }^{10} \mathrm{Be}(\mathrm{p}, \gamma){ }^{11} \mathrm{~B}$ & TALYS & 11.2278 \\
\hline${ }^{10} \mathrm{Be}(\mathrm{p}, \alpha){ }^{7} \mathrm{Li}$ & TALYS & 2.5626 & ${ }^{10} \mathrm{Be}(\alpha, n){ }^{13} \mathrm{C}$ & TALYS & 3.8353 \\
\hline${ }^{10} \mathrm{Be}(\mathrm{d}, \gamma){ }^{12} \mathrm{~B}$ & TALYS & 12.3735 & ${ }^{10} \mathrm{Be}(\mathrm{d}, \mathrm{n}){ }^{11} \mathrm{~B}$ & TALYS & 9.0032 \\
\hline${ }^{10} \mathrm{Be}(\mathrm{d}, \alpha){ }^{8} \mathrm{Li}$ & TALYS & 2.3707 & ${ }^{10} \mathrm{Be}\left({ }^{3} \mathrm{He}, \gamma\right){ }^{13} \mathrm{C}$ & TALYS & 24.4129 \\
\hline${ }^{10} \mathrm{Be}\left({ }^{3} \mathrm{He}, \mathrm{n}\right){ }^{12} \mathrm{C}$ & TALYS & 19.4666 & ${ }^{10} \mathrm{Be}\left({ }^{3} \mathrm{He}, \mathrm{p}\right){ }^{12} \mathrm{~B}$ & TALYS & 6.8800 \\
\hline${ }^{10} \mathrm{Be}\left({ }^{3} \mathrm{He}, \alpha\right){ }^{9} \mathrm{Be}$ & TALYS & 13.7654 & ${ }^{10} \mathrm{Be}(\mathrm{t}, \gamma){ }^{13} \mathrm{~B}$ & TALYS & 10.9943 \\
\hline${ }^{10} \mathrm{Be}(\mathrm{t}, \mathrm{n}){ }^{12} \mathrm{~B}$ & TALYS & 6.1163 & ${ }^{10} \mathrm{Be}(\mathrm{t}, \alpha){ }^{9} \mathrm{Li}$ & TALYS & 0.1773 \\
\hline${ }^{10} \mathrm{Be}(\mathrm{p}, \mathrm{t})^{4} \mathrm{He}$ & TALYS & 0.0960 & ${ }^{10} \mathrm{Be}\left({ }^{3} \mathrm{He}, \mathrm{d}\right){ }^{11} \mathrm{~B}$ & TALYS & 5.7343 \\
\hline${ }^{10} \mathrm{Be}\left({ }^{3} \mathrm{He}, \mathrm{t}\right){ }^{10} \mathrm{~B}$ & TALYS & 0.5374 & ${ }^{11} \mathrm{Be}(\mathrm{n}, \gamma){ }^{12} \mathrm{Be}$ & Rau94 & 3.1683 \\
\hline${ }^{11} \mathrm{Be}(\mathrm{p}, \alpha){ }^{8} \mathrm{Li}$ & TALYS & 4.0912 & ${ }^{11} \mathrm{Be}(\mathrm{p}, \mathrm{n}){ }^{11} \mathrm{~B}$ & TALYS & 10.7238 \\
\hline${ }^{11} \mathrm{Be}(\alpha, \mathrm{n}){ }^{14} \mathrm{C}$ & TALYS & 11.5077 & ${ }^{11} \mathrm{Be}(\alpha, \gamma){ }^{15} \mathrm{C}$ & TALYS & 12.7258 \\
\hline${ }^{11} \mathrm{Be}(\mathrm{d}, \gamma){ }^{13} \mathrm{~B}$ & TALYS & 16.7475 & ${ }^{11} \mathrm{Be}(\mathrm{d}, \mathrm{n}){ }^{12} \mathrm{~B}$ & TALYS & 11.8695 \\
\hline${ }^{11} \mathrm{Be}(\mathrm{d}, \mathrm{p})^{12} \mathrm{Be}$ & TALYS & 0.9438 & ${ }^{11} \mathrm{Be}(\mathrm{d}, \alpha)^{9} \mathrm{Li}$ & TALYS & 5.9305 \\
\hline${ }^{11} \mathrm{Be}\left({ }^{3} \mathrm{He}, \gamma\right){ }^{14} \mathrm{C}$ & TALYS & 32.0853 & ${ }^{11} \mathrm{Be}\left({ }^{3} \mathrm{He}, \mathrm{n}\right){ }^{13} \mathrm{C}$ & TALYS & 23.9089 \\
\hline${ }^{11} \mathrm{Be}\left({ }^{3} \mathrm{He}, \mathrm{p}\right){ }^{13} \mathrm{~B}$ & TALYS & 11.2540 & ${ }^{11} \mathrm{Be}\left({ }^{3} \mathrm{He}, \alpha\right){ }^{10} \mathrm{Be}$ & TALYS & 20.0736 \\
\hline${ }^{11} \mathrm{Be}(\mathrm{p}, \gamma){ }^{12} \mathrm{~B}$ & TALYS & 14.0941 & ${ }^{11} \mathrm{Be}(\mathrm{t}, \gamma){ }^{14} \mathrm{~B}$ & TALYS & 11.4598 \\
\hline${ }^{11} \mathrm{Be}(\mathrm{t}, \mathrm{n}){ }^{13} \mathrm{~B}$ & TALYS & 10.4903 & ${ }^{11} \mathrm{Be}(\mathrm{p}, \mathrm{t})^{9} \mathrm{Be}$ & TALYS & 1.1656 \\
\hline${ }^{11} \mathrm{Be}(\mathrm{p}, \mathrm{d}){ }^{10} \mathrm{Be}$ & TALYS & 1.7205 & ${ }^{12} \mathrm{Be}(\alpha, \gamma){ }^{16} \mathrm{C}$ & TALYS & 13.8079 \\
\hline${ }^{12} \mathrm{Be}(\alpha, \mathrm{n}){ }^{15} \mathrm{C}$ & TALYS & 9.5575 & ${ }^{12} \mathrm{Be}(\mathrm{d}, \gamma){ }^{14} \mathrm{~B}$ & TALYS & 14.5487 \\
\hline${ }^{12} \mathrm{Be}(\mathrm{d}, \mathrm{n}){ }^{13} \mathrm{~B}$ & TALYS & 13.5792 & ${ }^{12} \mathrm{Be}\left({ }^{3} \mathrm{He}, \gamma\right){ }^{15} \mathrm{C}$ & TALYS & 30.1351 \\
\hline${ }^{12} \mathrm{Be}\left({ }^{3} \mathrm{He}, \mathrm{n}\right){ }^{14} \mathrm{C}$ & TALYS & 28.9170 & ${ }^{12} \mathrm{Be}\left({ }^{3} \mathrm{He}, \mathrm{p}\right){ }^{14} \mathrm{~B}$ & TALYS & 9.0552 \\
\hline${ }^{12} \mathrm{Be}\left({ }^{3} \mathrm{He}, \alpha\right){ }^{11} \mathrm{Be}$ & TALYS & 17.4093 & ${ }^{12} \mathrm{Be}(\mathrm{p}, \gamma){ }^{13} \mathrm{~B}$ & TALYS & 15.8038 \\
\hline${ }^{12} \mathrm{Be}(\mathrm{p}, \mathrm{n}){ }^{12} \mathrm{~B}$ & TALYS & 10.9258 & ${ }^{12} \mathrm{Be}(\mathrm{t}, \gamma){ }^{15} \mathrm{~B}$ & TALYS & 11.0548 \\
\hline${ }^{12} \mathrm{Be}(\mathrm{t}, \mathrm{n}){ }^{14} \mathrm{~B}$ & TALYS & 8.2915 & ${ }^{12} \mathrm{Be}(\mathrm{p}, \alpha){ }^{9} \mathrm{Li}$ & TALYS & 4.9868 \\
\hline${ }^{12} \mathrm{Be}(\mathrm{p}, \mathrm{t}){ }^{10} \mathrm{Be}$ & TALYS & 4.8095 & ${ }^{8} \mathrm{~B}(\mathrm{p}, \gamma){ }^{9} \mathrm{C}$ & Des99Bea01 & 1.3000 \\
\hline${ }^{8} \mathrm{~B}(\alpha, \gamma){ }^{12} \mathrm{~N}$ & TALYS & 8.0083 & ${ }^{8} \mathrm{~B}(\mathrm{n}, \mathrm{p}){ }^{4} \mathrm{He}$ & TALYS & 18.8540 \\
\hline
\end{tabular}


TABle 4-Continued

\begin{tabular}{|c|c|c|c|c|c|}
\hline${ }^{8} \mathrm{~B}(\alpha, \mathrm{p}){ }^{11} \mathrm{C}$ & TALYS & 7.4071 & ${ }^{8} \mathrm{~B}(\mathrm{~d}, \gamma){ }^{10} \mathrm{C}$ & TALYS & 20.3585 \\
\hline${ }^{8} \mathrm{~B}\left({ }^{3} \mathrm{He}, \mathrm{p}\right){ }^{10} \mathrm{C}$ & TALYS & 14.8650 & ${ }^{8} \mathrm{~B}(\mathrm{t}, \gamma){ }^{11} \mathrm{C}$ & TALYS & 27.2210 \\
\hline${ }^{8} \mathrm{~B}(\mathrm{t}, \mathrm{n}){ }^{10} \mathrm{C}$ & TALYS & 14.1013 & ${ }^{8} \mathrm{~B}(\mathrm{t}, \mathrm{p}){ }^{10} \mathrm{~B}$ & TALYS & 18.5316 \\
\hline${ }^{8} \mathrm{~B}(\mathrm{t}, \alpha){ }^{7} \mathrm{Be}$ & TALYS & 19.6764 & ${ }^{8} \mathrm{~B}\left(\mathrm{n},{ }^{3} \mathrm{He}\right)^{6} \mathrm{Li}$ & TALYS & 1.9748 \\
\hline${ }^{8} \mathrm{~B}(\mathrm{n}, \mathrm{d}){ }^{7} \mathrm{Be}$ & TALYS & 2.0871 & ${ }^{8} \mathrm{~B}\left(\mathrm{~d},{ }^{3} \mathrm{He}\right){ }^{7} \mathrm{Be}$ & TALYS & 5.3560 \\
\hline${ }^{8} \mathrm{~B}\left(\mathrm{t},{ }^{3} \mathrm{He}\right){ }^{4} \mathrm{He}$ & TALYS & 18.0903 & ${ }^{10} \mathrm{~B}(\alpha, \mathrm{n}){ }^{13} \mathrm{~N}$ & CF88 & 1.0588 \\
\hline${ }^{10} \mathrm{~B}(\alpha, \gamma){ }^{14} \mathrm{~N}$ & TALYS & 11.6122 & ${ }^{10} \mathrm{~B}(\mathrm{n}, \gamma){ }^{11} \mathrm{~B}$ & TALYS & 11.4541 \\
\hline${ }^{10} \mathrm{~B}(\alpha, \mathrm{p}){ }^{13} \mathrm{C}$ & TALYS & 4.0616 & ${ }^{10} \mathrm{~B}(\mathrm{~d}, \gamma){ }^{12} \mathrm{C}$ & TALYS & 25.1864 \\
\hline${ }^{10} \mathrm{~B}(\mathrm{~d}, \mathrm{n}){ }^{11} \mathrm{C}$ & TALYs & 6.4648 & ${ }^{10} \mathrm{~B}(\mathrm{~d}, \mathrm{p}){ }^{11} \mathrm{~B}$ & TALYS & 9.2296 \\
\hline${ }^{10} \mathrm{~B}(\mathrm{~d}, \alpha){ }^{4} \mathrm{He}$ & TALYS & 17.9117 & ${ }^{10} \mathrm{~B}\left({ }^{3} \mathrm{He}, \gamma\right){ }^{13} \mathrm{~N}$ & TALYS & 21.6364 \\
\hline${ }^{10} \mathrm{~B}\left({ }^{3} \mathrm{He}, \mathrm{n}\right){ }^{12} \mathrm{~N}$ & TALYS & 1.5725 & ${ }^{10} \mathrm{~B}\left({ }^{3} \mathrm{He}, \mathrm{p}\right){ }^{12} \mathrm{C}$ & TALYS & 19.6929 \\
\hline${ }^{10} \mathrm{~B}(\mathrm{n}, \mathrm{p}){ }^{10} \mathrm{Be}$ & TALYs & 0.2263 & ${ }^{10} \mathrm{~B}(\mathrm{t}, \gamma){ }^{13} \mathrm{C}$ & TALYS & 23.8755 \\
\hline${ }^{10} \mathrm{~B}(\mathrm{t}, \mathrm{n}){ }^{12} \mathrm{C}$ & TALYS & 18.9292 & ${ }^{10} \mathrm{~B}(\mathrm{t}, \mathrm{p}){ }^{12} \mathrm{~B}$ & TALYS & 6.3426 \\
\hline${ }^{10} \mathrm{~B}(\mathrm{t}, \alpha){ }^{9} \mathrm{Be}$ & TALYS & 13.2280 & ${ }^{10} \mathrm{~B}(\mathrm{n}, \mathrm{t})^{4} \mathrm{He}$ & TALYS & 0.3224 \\
\hline${ }^{10} \mathrm{~B}(\alpha, \mathrm{d}){ }^{12} \mathrm{C}$ & TALYS & 1.3399 & ${ }^{10} \mathrm{~B}\left(\mathrm{p},{ }^{3} \mathrm{He}\right){ }^{4} \mathrm{He}$ & TALYS & -0.4414 \\
\hline${ }^{10} \mathrm{~B}(\mathrm{t}, \mathrm{d}){ }^{11} \mathrm{~B}$ & TALYS & 5.1969 & ${ }^{10} \mathrm{~B}\left({ }^{3} \mathrm{He}, \mathrm{d}\right){ }^{11} \mathrm{C}$ & TALYS & 959 \\
\hline${ }^{10} \mathrm{~B}(\mathrm{p}, \gamma){ }^{11} \mathrm{C}$ & NACREII & 8.6894 & ${ }^{10} \mathrm{~B}(\mathrm{p}, \alpha)^{7} \mathrm{Be}$ & NACREII & 1.1447 \\
\hline${ }^{11} \mathrm{~B}(\mathrm{p}, \mathrm{n}){ }^{11} \mathrm{C}$ & NACRE & -2.7647 & ${ }^{11} \mathbf{B}(\mathbf{n}, \gamma){ }^{12} \mathbf{B}$ & Rau94 & 3.3703 \\
\hline${ }^{11} \mathrm{~B}(\alpha, \mathrm{p}){ }^{14} \mathrm{C}$ & Wan91 & 0.7840 & ${ }^{11} \mathrm{~B}(\alpha, \gamma){ }^{15} \mathrm{~N}$ & Wan91 & 10.9914 \\
\hline${ }^{11} \mathrm{~B}(\mathrm{~d}, \gamma){ }^{13} \mathrm{C}$ & TALYS & 18.6786 & ${ }^{11} \mathbf{B}(\mathbf{d}, \mathbf{n}){ }^{12} \mathbf{C}$ & TALYS & 13.7323 \\
\hline${ }^{11} \mathbf{B}(\mathbf{d}, \mathbf{p}){ }^{12} \mathbf{B}$ & TALYS & 1.1458 & ${ }^{11} \mathrm{~B}(\mathrm{~d}, \alpha){ }^{9} \mathrm{Be}$ & TALYS & 8.0311 \\
\hline${ }^{11} \mathrm{~B}\left({ }^{3} \mathrm{He}, \gamma\right){ }^{14} \mathrm{~N}$ & TALYS & 20.7357 & ${ }^{11} \mathrm{~B}\left({ }^{3} \mathrm{He}, \mathrm{n}\right){ }^{13} \mathrm{~N}$ & TALYS & 10.1823 \\
\hline${ }^{11} \mathrm{~B}\left({ }^{3} \mathrm{He}, \mathrm{p}\right){ }^{13} \mathrm{C}$ & TALYS & 13.1851 & ${ }^{11} \mathrm{~B}\left({ }^{3} \mathrm{He}, \alpha\right){ }^{10} \mathrm{~B}$ & $\mathrm{TA}$ & 9.1235 \\
\hline${ }^{11} \mathrm{~B}(\mathrm{t}, \gamma){ }^{14} \mathrm{C}$ & TALYS & 20. & ${ }^{11} \mathrm{~B}(\mathrm{t}, \mathrm{n}){ }^{13} \mathrm{C}$ & TA & 12.4214 \\
\hline${ }^{11} \mathrm{~B}(\mathrm{t}, \alpha)^{10} \mathrm{Be}$ & TALYS & 8.5861 & ${ }^{11} \mathrm{~B}(\mathrm{t}, \mathrm{p}){ }^{13} \mathrm{~B}$ & TALYS & -0.2335 \\
\hline${ }^{11} \mathrm{~B}\left({ }^{3} \mathrm{He}, \mathrm{d}\right){ }^{12} \mathrm{C}$ & TALYS & 10.4634 & ${ }^{11} \mathrm{~B}(\mathrm{p}, \gamma){ }^{12} \mathrm{C}$ & NACREII & 15.9569 \\
\hline${ }^{11} \mathrm{~B}(\mathrm{p}, \alpha){ }^{4} \mathrm{He}$ & NACREII & 8.6 & ${ }^{11} \mathrm{~B}(\alpha, \mathrm{n}){ }^{14} \mathrm{~N}$ & NACREII & 0.1581 \\
\hline${ }^{12} \mathrm{~B}(\mathrm{p}, \alpha)^{9} \mathrm{Be}$ & TALYS & 6.8 & ${ }^{12} \mathrm{~B}(\alpha, \gamma){ }^{16} \mathrm{~N}$ & TALYS & 10.1101 \\
\hline${ }^{12} \mathrm{~B}(\mathrm{p}, \mathrm{n}){ }^{12} \mathrm{C}$ & TALYS & 12.5866 & ${ }^{12} \mathrm{~B}(\alpha, \mathrm{n})^{15} \mathrm{~N}$ & TALYS & 7.6211 \\
\hline${ }^{12} \mathrm{~B}(\mathrm{~d}, \gamma){ }^{14} \mathrm{C}$ & TALYS & 23.4847 & ${ }^{12} \mathrm{~B}(\mathrm{~d}, \mathrm{n}){ }^{13} \mathrm{C}$ & TALYS & 15.3083 \\
\hline${ }^{12} \mathrm{~B}(\mathrm{~d}, \mathrm{p}){ }^{13} \mathrm{~B}$ & TA & 2.6535 & ${ }^{12} \mathrm{~B}(\mathrm{~d}, \alpha){ }^{10} \mathrm{Be}$ & YS & 11.4730 \\
\hline${ }^{12} \mathrm{~B}\left({ }^{3} \mathrm{He}, \gamma\right){ }^{15} \mathrm{~N}$ & TALYS & 28.1987 & ${ }^{12} \mathrm{~B}\left({ }^{3} \mathrm{He}, \mathrm{n}\right){ }^{14} \mathrm{~N}$ & TALYS & 17.3654 \\
\hline${ }^{12} \mathrm{~B}\left({ }^{3} \mathrm{He}, \mathrm{p}\right){ }^{14} \mathrm{C}$ & TALYS & 17.9913 & ${ }^{12} \mathrm{~B}\left({ }^{3} \mathrm{He}, \alpha\right)^{11} \mathrm{~B}$ & TALYS & 17.2073 \\
\hline${ }^{12} \mathrm{~B}(\mathrm{n}, \gamma){ }^{13} \mathrm{~B}$ & TALYS & 4.8780 & ${ }^{12} \mathrm{~B}(\mathrm{p}, \gamma){ }^{13} \mathrm{C}$ & TALYS & 17.5329 \\
\hline${ }^{12} \mathrm{~B}(\mathrm{t}, \gamma){ }^{15} \mathrm{C}$ & TALYS & 18.4456 & ${ }^{12} \mathrm{~B}(\mathrm{t}, \mathrm{n}){ }^{14} \mathrm{C}$ & TALYS & 17.2275 \\
\hline${ }^{12} \mathrm{~B}(\mathrm{t}, \alpha)^{11} \mathrm{Be}$ & TALYS & 5.7198 & ${ }^{9} \mathrm{C}(\alpha, \mathrm{p}){ }^{12} \mathrm{~N}$ & Wie89 & 6.7083 \\
\hline${ }^{9} \mathrm{C}(\alpha, \gamma){ }^{13} \mathrm{O}$ & TALYS & 8.2234 & ${ }^{9} \mathrm{C}(\mathrm{d}, \mathrm{p}){ }^{10} \mathrm{C}$ & TALYS & 19.0586 \\
\hline${ }^{9} \mathrm{C}(\mathrm{n}, \gamma){ }^{10} \mathrm{C}$ & TALYS & 21.2831 & ${ }^{9} \mathrm{C}(\mathrm{t}, \gamma){ }^{12} \mathrm{~N}$ & TALYS & 26.5222 \\
\hline${ }^{9} \mathrm{C}(\mathrm{t}, \mathrm{p}){ }^{11} \mathrm{C}$ & TALYS & 25.9210 & ${ }^{9} \mathrm{C}(\mathrm{t}, \alpha){ }^{8} \mathrm{~B}$ & TALYS & 18.5139 \\
\hline${ }^{9} \mathrm{C}\left(\mathrm{n},{ }^{3} \mathrm{He}\right){ }^{7} \mathrm{Be}$ & TALYS & 6.2806 & ${ }^{9} \mathrm{C}(\mathrm{n}, \mathrm{d}){ }^{8} \mathrm{~B}$ & TALYS & 0.9246 \\
\hline${ }^{11} \mathrm{C}(\mathrm{p}, \gamma){ }^{12} \mathrm{~N}$ & Tan03 & 0.6012 & ${ }^{11} \mathrm{C}(\mathrm{n}, \gamma){ }^{12} \mathrm{C}$ & Rau94 & 18.7216 \\
\hline${ }^{11} \mathbf{C}(\mathbf{n}, \alpha){ }^{4} \mathbf{H e}$ & Rau94 & 11.4469 & $\left.{ }^{11} \mathrm{C}(\alpha, \mathrm{p})\right)^{14} \mathrm{~N}$ & NACRE & 2.9228 \\
\hline${ }^{11} \mathrm{C}(\mathrm{d}, \gamma){ }^{13} \mathrm{~N}$ & TALYS & 18.4405 & ${ }^{11} \mathbf{C}(\mathbf{d}, \mathbf{p}){ }^{12} \mathbf{C}$ & TALYS & 16.4971 \\
\hline${ }^{11} \mathrm{C}\left({ }^{3} \mathrm{He}, \gamma\right){ }^{14} \mathrm{O}$ & TALYS & 17.5742 & ${ }^{11} \mathrm{C}\left({ }^{3} \mathrm{He}, \mathrm{p}\right){ }^{13} \mathrm{~N}$ & TALYS & 12.9471 \\
\hline
\end{tabular}


TABLE 4-Continued

\begin{tabular}{|c|c|c|c|c|c|}
\hline${ }^{11} \mathrm{C}\left({ }^{3} \mathrm{He}, \alpha\right){ }^{10} \mathrm{C}$ & TALYS & 7.4579 & ${ }^{11} \mathrm{C}(\mathrm{t}, \gamma){ }^{14} \mathrm{~N}$ & TALYS & 22.7367 \\
\hline${ }^{11} \mathrm{C}(\mathrm{t}, \mathrm{n}){ }^{13} \mathrm{~N}$ & TALYS & 12.1833 & ${ }^{11} \mathrm{C}(\mathrm{t}, \mathrm{p}){ }^{13} \mathrm{C}$ & TALYS & 15.1861 \\
\hline${ }^{11} \mathrm{C}(\mathrm{t}, \alpha){ }^{10} \mathrm{~B}$ & TALYS & 11.1245 & ${ }^{11} \mathrm{C}(\alpha, \gamma){ }^{15} \mathrm{O}$ & TALYS & 10.2196 \\
\hline${ }^{11} \mathrm{C}(\mathrm{t}, \mathrm{d}){ }^{12} \mathrm{C}$ & TALYS & 12.4644 & ${ }^{11} \mathrm{C}\left(\mathrm{t},{ }^{3} \mathrm{He}\right){ }^{11} \mathrm{~B}$ & TALYS & 2.0010 \\
\hline${ }^{12} \mathrm{C}(\alpha, \gamma){ }^{16} \mathrm{O}$ & NACREII & 7.1619 & ${ }^{12} \mathrm{C}(\mathrm{d}, \gamma){ }^{14} \mathrm{~N}$ & TALYS & 10.2723 \\
\hline${ }^{12} \mathrm{C}(\mathrm{d}, \mathrm{p}){ }^{13} \mathrm{C}$ & TALYS & 2.7217 & ${ }^{12} \mathrm{C}\left({ }^{3} \mathrm{He}, \gamma\right){ }^{15} \mathrm{O}$ & TALYS & 12.0756 \\
\hline${ }^{12} \mathrm{C}\left({ }^{3} \mathrm{He}, \mathrm{p}\right){ }^{14} \mathrm{~N}$ & TALYS & 4.7788 & ${ }^{12} \mathrm{C}\left({ }^{3} \mathrm{He}, \alpha\right){ }^{11} \mathrm{C}$ & TALYS & 1.8560 \\
\hline${ }^{12} \mathrm{C}(\mathrm{n}, \gamma){ }^{13} \mathrm{C}$ & TALYS & 4.9463 & ${ }^{12} \mathrm{C}(\mathrm{p}, \gamma){ }^{13} \mathrm{~N}$ & NACREII & 1.9435 \\
\hline${ }^{12} \mathrm{C}(\mathrm{t}, \gamma){ }^{15} \mathrm{~N}$ & TALYS & 14.8484 & ${ }^{12} \mathrm{C}(\mathrm{t}, \mathrm{n}){ }^{14} \mathrm{~N}$ & TALYS & 4.0151 \\
\hline${ }^{12} \mathrm{C}(\mathrm{t}, \mathrm{p}){ }^{14} \mathrm{C}$ & TALYS & 4.6409 & ${ }^{12} \mathrm{C}(\mathrm{t}, \alpha)^{11} \mathrm{~B}$ & TALYS & 3.8570 \\
\hline${ }^{13} \mathrm{C}(\alpha, \gamma){ }^{17} \mathrm{O}$ & TALYS & 6.3587 & ${ }^{13} \mathrm{C}(\mathrm{d}, \gamma){ }^{15} \mathrm{~N}$ & TALYS & 16.1593 \\
\hline${ }^{13} \mathrm{C}(\mathrm{d}, \mathrm{n}){ }^{14} \mathrm{~N}$ & TALYS & 5.3260 & ${ }^{13} \mathrm{C}(\mathrm{d}, \mathrm{p}){ }^{14} \mathrm{C}$ & TALYS & 5.9519 \\
\hline${ }^{13} \mathrm{C}(\mathrm{d}, \alpha){ }^{11} \mathrm{~B}$ & TALYS & 5.1679 & ${ }^{13} \mathrm{C}\left({ }^{3} \mathrm{He}, \gamma\right){ }^{16} \mathrm{O}$ & TALYS & 22.7932 \\
\hline${ }^{13} \mathrm{C}\left({ }^{3} \mathrm{He}, \mathrm{n}\right){ }^{15} \mathrm{O}$ & TALYS & 7.1293 & ${ }^{13} \mathrm{C}\left({ }^{3} \mathrm{He}, \mathrm{p}\right){ }^{15} \mathrm{~N}$ & TALYS & 10.6658 \\
\hline${ }^{13} \mathrm{C}\left({ }^{3} \mathrm{He}, \alpha\right){ }^{12} \mathrm{C}$ & TALYS & 15.6313 & ${ }^{13} \mathrm{C}(\mathrm{n}, \gamma){ }^{14} \mathrm{C}$ & TALYS & 8.1764 \\
\hline${ }^{13} \mathrm{C}(\mathrm{p}, \gamma){ }^{14} \mathrm{~N}$ & NACREII & 7.5506 & ${ }^{13} \mathrm{C}(\mathrm{t}, \gamma){ }^{16} \mathrm{~N}$ & TALYS & 12.3911 \\
\hline${ }^{13} \mathrm{C}(\mathrm{t}, \mathrm{n}){ }^{15} \mathrm{~N}$ & TALYS & 9.9021 & ${ }^{13} \mathrm{C}(\mathrm{t}, \mathrm{p}){ }^{15} \mathrm{C}$ & TALYS & 0.9127 \\
\hline${ }^{13} \mathrm{C}(\mathrm{t}, \alpha){ }^{12} \mathrm{~B}$ & TALYS & 2.2810 & ${ }^{13} \mathrm{C}(\alpha, \mathrm{n}){ }^{16} \mathrm{O}$ & NACREII & 2.2156 \\
\hline${ }^{14} \mathrm{C}(\mathrm{d}, \mathrm{n}){ }^{15} \mathrm{~N}$ & Kaw91 & 7.9829 & ${ }^{14} \mathrm{C}(\mathrm{n}, \gamma){ }^{15} \mathrm{C}$ & Kaw91 & 1.2181 \\
\hline${ }^{14} \mathrm{C}(\alpha, \gamma){ }^{18} \mathrm{O}$ & ILCCF10 & 6.2263 & ${ }^{14} \mathrm{C}(\mathrm{p}, \gamma){ }^{15} \mathrm{~N}$ & ILCCF10 & 10.2074 \\
\hline${ }^{14} \mathrm{C}(\mathrm{d}, \gamma){ }^{16} \mathrm{~N}$ & TALYS & 10.4719 & ${ }^{14} \mathrm{C}(\mathrm{d}, \alpha){ }^{12} \mathrm{~B}$ & TALYS & 0.3618 \\
\hline${ }^{14} \mathrm{C}\left({ }^{3} \mathrm{He}, \gamma\right){ }^{17} \mathrm{O}$ & TALYS & 18.7599 & ${ }^{14} \mathrm{C}\left({ }^{3} \mathrm{He}, \mathrm{n}\right){ }^{16} \mathrm{O}$ & TALYS & 14.6168 \\
\hline${ }^{14} \mathrm{C}\left({ }^{3} \mathrm{He}, \mathrm{p}\right){ }^{16} \mathrm{~N}$ & TALYS & 4.9784 & ${ }^{14} \mathrm{C}\left({ }^{3} \mathrm{He}, \alpha\right){ }^{13} \mathrm{C}$ & TALYS & 12.4012 \\
\hline${ }^{14} \mathrm{C}(\mathrm{t}, \gamma){ }^{17} \mathrm{~N}$ & TALYS & 10.0987 & ${ }^{14} \mathrm{C}(\mathrm{t}, \mathrm{n}){ }^{16} \mathrm{~N}$ & TALYS & 4.2147 \\
\hline${ }^{15} \mathrm{C}(\alpha, \gamma){ }^{19} \mathrm{O}$ & TALYS & 8.9631 & ${ }^{15} \mathrm{C}(\alpha, \mathrm{n})^{18} \mathrm{O}$ & TALYS & 5.0082 \\
\hline${ }^{15} \mathrm{C}(\mathrm{n}, \gamma){ }^{16} \mathrm{C}$ & TALYS & 4.2504 & ${ }^{15} \mathrm{C}(\mathrm{p}, \gamma){ }^{16} \mathrm{~N}$ & TALYS & 11.4784 \\
\hline${ }^{15} \mathrm{C}(\mathrm{p}, \mathrm{n}){ }^{15} \mathrm{~N}$ & TALYS & 8.9893 & $\left.{ }^{15} \mathrm{C}(\mathrm{p}, \alpha)\right)^{12} \mathrm{~B}$ & TALYS & 1.3683 \\
\hline${ }^{15} \mathrm{C}(\mathrm{p}, \mathrm{d}){ }^{14} \mathrm{C}$ & TALYS & 1.0065 & ${ }^{12} \mathrm{~N}(\mathrm{n}, \mathrm{p}){ }^{12} \mathrm{C}$ & TALYS & 18.1204 \\
\hline${ }^{12} \mathrm{~N}(\alpha, \mathrm{p}){ }^{15} \mathrm{O}$ & TALYS & 9.6184 & ${ }^{12} \mathrm{~N}(\mathrm{n}, \gamma)^{13} \mathrm{~N}$ & TALYS & 20.0639 \\
\hline${ }^{12} \mathrm{~N}(\mathrm{p}, \gamma){ }^{13} \mathrm{O}$ & TALYS & 1.5151 & ${ }^{12} \mathrm{~N}(\mathrm{n}, \mathrm{d}){ }^{11} \mathrm{C}$ & TALYS & 1.6234 \\
\hline${ }^{13} \mathrm{~N}(\mathrm{n}, \gamma){ }^{14} \mathrm{~N}$ & TALYS & 10.5534 & ${ }^{13} \mathrm{~N}(\alpha, \gamma){ }^{17} \mathrm{~F}$ & TALYS & 5.8187 \\
\hline${ }^{13} \mathrm{~N}(\mathrm{n}, \mathrm{p}){ }^{13} \mathrm{C}$ & TALYS & 3.0028 & ${ }^{13} \mathrm{~N}(\mathrm{p}, \gamma){ }^{14} \mathrm{O}$ & NACREII & 4.6271 \\
\hline${ }^{13} \mathrm{~N}(\mathrm{n}, \mathrm{d}){ }^{12} \mathrm{C}$ & TALYS & 0.2811 & ${ }^{14} \mathrm{~N}(\mathrm{n}, \mathrm{p}){ }^{14} \mathrm{C}$ & CF88 & 0.6259 \\
\hline${ }^{14} \mathrm{~N}(\alpha, \gamma){ }^{18} \mathrm{~F}$ & ILCCF10 & 4.4146 & ${ }^{14} \mathrm{~N}(\mathrm{n}, \gamma){ }^{15} \mathrm{~N}$ & TALYS & 10.8333 \\
\hline${ }^{14} \mathrm{~N}(\mathrm{p}, \gamma){ }^{15} \mathrm{O}$ & NACREII & 7.2968 & ${ }^{15} \mathrm{~N}(\alpha, \gamma){ }^{19} \mathrm{~F}$ & ILCCF10 & 4.0137 \\
\hline${ }^{15} \mathrm{~N}(\mathrm{n}, \gamma){ }^{16} \mathrm{~N}$ & TALYS & 2.4891 & ${ }^{15} \mathrm{~N}(\mathrm{p}, \gamma){ }^{16} \mathrm{O}$ & NACREII & 12.1274 \\
\hline${ }^{15} \mathrm{~N}(\mathrm{p}, \alpha){ }^{12} \mathrm{C}$ & NACREII & 4.9655 & ${ }^{14} \mathrm{O}(\mathrm{n}, \mathrm{p}){ }^{14} \mathrm{~N}$ & CF88 & 5.9263 \\
\hline${ }^{14} \mathrm{O}(\alpha, \gamma){ }^{18} \mathrm{Ne}$ & Wie87 & 5.1151 & ${ }^{14} \mathrm{O}(\alpha, \mathrm{p}){ }^{17} \mathrm{~F}$ & Bar97C & 1.1916 \\
\hline${ }^{14} \mathrm{O}(\mathrm{n}, \gamma){ }^{15} \mathrm{O}$ & TALYS & 13.2231 & ${ }^{14} \mathrm{O}(\mathrm{n}, \alpha)^{11} \mathrm{C}$ & TALYS & 3.0035 \\
\hline${ }^{14} \mathrm{O}\left(\mathrm{n},{ }^{3} \mathrm{He}\right){ }^{12} \mathrm{C}$ & TALYS & 1.1475 & ${ }^{15} \mathrm{O}(\alpha, \gamma){ }^{19} \mathrm{Ne}$ & ILCCF10 & 3.5291 \\
\hline${ }^{15} \mathrm{O}(\mathrm{n}, \gamma){ }^{16} \mathrm{O}$ & TALYS & 15.6639 & ${ }^{15} \mathrm{O}(\mathrm{n}, \mathrm{p}){ }^{15} \mathrm{~N}$ & TALYS & 3.5365 \\
\hline${ }^{15} \mathrm{O}(\mathrm{n}, \alpha)^{12} \mathrm{C}$ & TALYS & 8.5020 & ${ }^{16} \mathrm{O}(\mathrm{n}, \gamma){ }^{17} \mathrm{O}$ & Iga95 & 4.1431 \\
\hline${ }^{16} \mathrm{O}(\mathrm{p}, \alpha){ }^{13} \mathrm{~N}$ & CF88 & $-5.2184 \&{ }^{16} \mathrm{O}(\mathrm{p}, \gamma){ }^{17} \mathrm{~F}$ & ILCCF10 & 0.6003 & \\
\hline${ }^{16} \mathrm{O}(\alpha, \gamma){ }^{20} \mathrm{Ne}$ & ILCCF10 & 4.7298 & ${ }^{17} \mathrm{O}(\mathrm{n}, \alpha){ }^{14} \mathrm{C}$ & Koe91 & 1.8177 \\
\hline
\end{tabular}




\begin{tabular}{|c|c|c|c|c|c|}
\hline \multirow[b]{2}{*}{${ }^{17} \mathrm{O}(\mathrm{n}, \gamma){ }^{18} \mathrm{O}$} & \multirow[b]{2}{*}{ TALYS } & \multicolumn{4}{|c|}{ TABLE 4-Continued } \\
\hline & & 8.0440 & ${ }^{17} \mathrm{O}(\mathrm{p}, \gamma){ }^{18} \mathrm{~F}$ & ILCCF10 & 5.6065 \\
\hline${ }^{17} \mathrm{O}(\mathrm{p}, \alpha){ }^{14} \mathrm{~N}$ & ILCCF10 & 1.1918 & ${ }^{17} \mathrm{O}(\alpha, \gamma){ }^{21} \mathrm{Ne}$ & CF88 & 7.3479 \\
\hline${ }^{17} \mathrm{O}(\alpha, \mathrm{n})^{20} \mathrm{Ne}$ & NACRE & 0.5867 & ${ }^{18} \mathrm{O}(\mathrm{n}, \gamma){ }^{19} \mathrm{O}$ & TALYS & 3.9549 \\
\hline${ }^{18} \mathrm{O}(\mathrm{p}, \gamma){ }^{19} \mathrm{~F}$ & ILCCF10 & 7.9949 & ${ }^{18} \mathrm{O}(\alpha, \gamma){ }^{22} \mathrm{Ne}$ & ILCCF 10 & 9.6681 \\
\hline${ }^{18} \mathrm{O}(\mathrm{p}, \alpha){ }^{15} \mathrm{~N}$ & ILCCF10 & 3.9811 & ${ }^{19} \mathrm{O}(\alpha, \gamma){ }^{23} \mathrm{Ne}$ & TALYS & 10.9139 \\
\hline${ }^{19} \mathrm{O}(\alpha, \mathrm{n}){ }^{22} \mathrm{Ne}$ & TALYS & 5.7132 & ${ }^{19} \mathrm{O}(\mathrm{n}, \gamma){ }^{20} \mathrm{O}$ & TALYS & 7.6087 \\
\hline${ }^{19} \mathrm{O}(\mathrm{p}, \gamma){ }^{20} \mathrm{~F}$ & TALYS & 10.6413 & ${ }^{19} \mathrm{O}(\mathrm{p}, \mathrm{n}){ }^{19} \mathrm{~F}$ & TALYS & 4.0399 \\
\hline${ }^{19} \mathrm{O}(\mathrm{p}, \alpha){ }^{16} \mathrm{~N}$ & TALYS & 2.5153 & ${ }^{17} \mathrm{~F}(\mathrm{n}, \alpha){ }^{14} \mathrm{~N}$ & NACRE & 4.7347 \\
\hline${ }^{17} \mathrm{~F}(\mathrm{n}, \mathrm{p}){ }^{17} \mathrm{O}$ & TALYS & 3.5429 & ${ }^{17} \mathrm{~F}(\mathrm{p}, \gamma){ }^{18} \mathrm{Ne}$ & ILCCF10 & 3.9235 \\
\hline${ }^{17} \mathrm{~F}(\alpha, \gamma){ }^{21} \mathrm{Na}$ & TALYS & 6.5608 & ${ }^{17} \mathrm{~F}(\alpha, \mathrm{p}){ }^{20} \mathrm{Ne}$ & TALYS & 4.1296 \\
\hline${ }^{17} \mathrm{~F}(\mathrm{n}, \gamma){ }^{18} \mathrm{~F}$ & TALYS & 9.1493 & ${ }^{18} \mathrm{~F}(\mathrm{n}, \alpha){ }^{15} \mathrm{~N}$ & CF88 & 6.4187 \\
\hline${ }^{18} \mathrm{~F}(\mathrm{n}, \mathrm{p}){ }^{18} \mathrm{O}$ & TALYS & 2.4375 & ${ }^{18} \mathrm{~F}(\mathrm{p}, \gamma){ }^{19} \mathrm{Ne}$ & ILCCF10 & 6.4112 \\
\hline${ }^{18} \mathrm{~F}(\mathrm{p}, \alpha){ }^{15} \mathrm{O}$ & ILCCF10 & 2.8822 & ${ }^{18} \mathrm{~F}(\alpha, \gamma){ }^{22} \mathrm{Na}$ & TALYS & 8.4810 \\
\hline${ }^{18} \mathrm{~F}(\alpha, \mathrm{p}){ }^{21} \mathrm{Ne}$ & TALYS & 1.7414 & ${ }^{18} \mathrm{~F}(\mathrm{n}, \gamma){ }^{19} \mathrm{~F}$ & TALYS & 10.4324 \\
\hline${ }^{19} \mathrm{~F}(\alpha, \gamma){ }^{23} \mathrm{Na}$ & TALYS & 10.4674 & ${ }^{19} \mathrm{~F}(\alpha, \mathrm{p}){ }^{22} \mathrm{Ne}$ & TALYS & 1.6733 \\
\hline${ }^{19} \mathrm{~F}(\mathrm{n}, \gamma){ }^{20} \mathrm{~F}$ & TALYS & 6.6013 & ${ }^{19} \mathrm{~F}(\mathrm{p}, \gamma){ }^{20} \mathrm{Ne}$ & NACRE & 12.8435 \\
\hline${ }^{19} \mathrm{~F}(\mathrm{p}, \alpha){ }^{16} \mathrm{O}$ & NACRE & 8.1137 & ${ }^{18} \mathrm{Ne}(\mathrm{n}, \alpha){ }^{15} \mathrm{O}$ & TALYS & 8.1080 \\
\hline${ }^{18} \mathrm{Ne}(\mathrm{n}, \mathrm{p}){ }^{18} \mathrm{~F}$ & TALYS & 5.2258 & ${ }^{19} \mathrm{Ne}(\mathrm{n}, \mathrm{p}){ }^{19} \mathrm{~F}$ & TALYS & 4.0212 \\
\hline${ }^{19} \mathrm{Ne}(\mathrm{p}, \gamma){ }^{20} \mathrm{Na}$ & ILCCF10 & 2.1924 & ${ }^{19} \mathrm{Ne}(\mathrm{n}, \alpha){ }^{16} \mathrm{O}$ & TALYS & 12.1348 \\
\hline${ }^{20} \mathrm{Na}(\mathrm{n}, \alpha){ }^{17} \mathrm{~F}$ & TALYS & 10.5427 & & & \\
\hline
\end{tabular}

References: NACRE $(\overline{\text { Angulo et al. }} \mid \overline{1999})$, NACRE II $(\overline{\mathrm{Xu} \text { et al. }} \mid \overline{2010}, \overline{2011})$,

DAACV04 (Descouvemont et al. 2004), ILCCF10 (Iliadis et al. 2010), CF88 (Caughlan \& Fowler 1988), MF89 (Malaney \& Fowler 1989), Boy93 (Boyd et al.|1993), Bal95 (Balbes et al. 1995), Hei98 (Heil et al. 1998), Rau94 (Rauscher et al. 1994), Des99 (Descouvemont 1999), Bea01 (Beaumel et al. 2001),

Tan03 (Tang et al. 2003), Wan91 (Wang et al. 1991), Efr96 (Efros et al. 1996), Wie87 (Wiescher et al. 1987), Bar97 (Bardavan \& Smith 1997), Koe91 (Koehler \& Graff|1991), Cam08 (Camargo et al.|2008), And06 (Ando et al. 2006), Ser04 (Serpico et al. 2004), Wag69 (Wagoner 1969), Has09 (Hashimoto et al. 2009), Wie89 (Wiescher et al. 1989), FK90 (Fukugita \& Kaiino 1990), Bru91 (Brune et al.|1991),

Bec92 (Becchetti et al. 1992), Iga95 (Igashira et al. 1995), Cyb08 (Cyburt \& Davids 2008), Miz00 (Mizoi et al. 2000).

Reaction and references shown in bold face : re-evaluated rate for the improved network, see text. 


\section{REFERENCES}

Ames, O., Owen, G. E., \& Swartz, C. D. 1957, Phys. Rev., 106, 775

Ando, S., Cyburt, R. H., Hong, S. W., \& Hyun, C. H. 2006, Phys. Rev. C, 74, 025809

Angulo, C. m, Arnould, M. , Rayet, M. , Descouvemont, P. , Baye, D. , Leclerq-Willain, C. , Coc, A. , Barhoumi, S. ,Aguer, P. , Rolfs, C. , Kunz, R. , Hammer, W. , Mayer, A. , Paradellis, T. , Kossionides, S. , Chronidou,C. , Spyrou, K. , Degl'Innocenti, S. , Fiorentini, G. , Ricci, B. , Zavatarelli, D. Providencia, C. , Wolters, H. , Soares, J. , Grama, C. , Rahighi, J. , Shotter, A. , Lamehi Rachti, M. Nucl. Phys. A, 656 (1999) 3-183 and http://pntpm.ulb.ac.be/nacre.htm.

Angulo, C., Casarejos, E., Coude, M., Demaret, P., Leleux, P., Vanderbist, F., Coc, A., Kiener, J., Tatischeff, V., Davinson, T., Murphy, A.S., Achouri, N.L.,Orr, N.A., Cortina-Gil, D., Figuera, P., Fulton, B.R., Mukha, I. and, E. 2005, ApJ, 630, L105 astro-ph/0508454.

Audi, G., Bersillon, O., Blachot, J. \& Wapstra, A. H. 2003 Nucl. Phys. A, 729, 3

Asplund, M., Lambert, D. L., Nissen, P. E., Primas, F., \& Smith, V. V. 2006, ApJ, 644, 229

Aver, E., Olive, K. A., \& Skillman, E. D. 2010, J. Cosmology Astropart. Phys., 05, 003

Balbes, M. J., Farrell, M. M., Boyd, R. N., Gu, X., Hencheck, M., Kalen, J. D., Mitchell, C. A., Kolata, J. J., Lamkin, K., Smith, R., Tighe, R., Ashktorab, K., Becchetti, F. D., Brown, J., Roberts, D., Wang, T.-F., Humphrey, D., Vourvopoulos, G., Islam, M. S. 1995, Nucl. Phys. A, 584,315

Bania T., Rood R. and Balser D. 2002 Nature, 415 54

Bardayan, D. W., \& Smith, M. S. 1997, Phys. Rev. C, 56, 1647

Barhoumi, S., et al. 1991, Nuclear Physics A, 535, 107
Beaumel, D., Kubo, T., Teranishi, T., Sakurai, H., Fortier, S., Mengoni, A., Aoi, N., Fukuda, N., Hirai, M., Imai, N., Iwasaki, H., Kumagai, H., Laurent, H., Lukyanov, S. M., Maison, J. M., Motobayashi, T., Nakamura, T., Ohnuma, H., Pita, S., Yoneda, K., Ishihara, M. 2001, Physics Letters B, 514, 226

Becchetti, F. D., Brown, J. A., Liu, W. Z., Jnecke, J. W., Roberts, D. A., Kolata, J. J., Smith, R. J., Lamkin, K., Morsad, A., Warner, R. E., Boyd, R. N., Kalen, J. D., 1992, Nuclear Physics A, 550, 507

Boesgaard, A.M., et al 2010 Proceedings IAU Symposium No. 268, Light Elements in the Universe 9-13 November, Geneva, Switzerland, Eds. Corinne Charbonnel, Monica Tosi, Francesca Primas \& Christina Chiappini Cambridge University Press, p. 231

Boyd, R. N., \& Kajino, T. 1989, ApJ, 336, L55

Boyd, R.N., Mitchell, C.A., \& Meyer, B.S. 1993, Phys. Rev. C, 47, 2369

Boyd, R.N., Brune, C.R., Fuller, G.M. and Smith, C.J. 2010, Phys. Rev. D, 82, 105005.

Brune, C. R., Kavanagh, R. W., Kellogg, S. E., \& Wang, T. R. 1991, Phys. Rev. C, 43, 875

Camargo, O., Guimarães, V., Lichtenthäler, R., Scarduelli, V., Kolata, J. J., Bertulani, C. A., Amro, H., Becchetti, F. D., Jiang, Hao, Aguilera, E. F., Lizcano, D., Martinez-Quiroz, E., Garcia, H. 2008, Phys. Rev. C, 78, 034605

Capote, R., Herman, M., Oblozinsky, P., Young, P. G., Goriely, S., Belgya, T., Ignatyuk, A. V., Koning, A. J., Hilaire, S., Plujko, V. A., Avrigeanu, M., Bersillon, O., Chadwick, M. B., Fukahori, T., Ge, Z. G., Han, Y. L., Kailas, S., Kopecky, J., Maslov, V. M., Reffo, G., Sin, M., Soukhovitskii, E. S., \& Talou, P. 2009, Nuclear Data Sheets, 110, 3107

Cassisi, S., \& Castellani, V. 1993, ApJS, 88, 509

Caughlan, G. R., \& Fowler, W. A. 1988, Atomic Data and Nuclear Data Tables, 40, 283

Cayrel, R., et al. 2007, A\&A, 473, L37 
Chakraborty, N., Fields, B. D., \& Olive, K. A. 2011, Phys. Rev. D, 83, 063006

Class, C. M., Price, J. E., \& Risser, J. R. 1965, Nucl. Phys. A, 71, 433

Coc, A., Vangioni-Flam, E., Descouvemont, P., Adahchour, A., \& Angulo, C. 2004, ApJ, 600, 544 astro-ph/0309480.

Coc, A., Nunes, N. J., Olive, K. A., Uzan, J.-P., \& Vangioni, E. 2007, Phys. Rev. D, 76, 023511 astro-ph/0610733.

Coc, A., \& Vangioni, E. 2010, Journal of Physics Conference Series, 202, 012001

La Cognata, M., Del Zoppo, A., Figuera, P., Musumarra, A., Alba, R., et al. 2008, PhLB, 664,157

La Cognata, M., Del Zoppo, A., Alba, R., Cherubini, S., Colonna, N., et al. 2009, ApJ, 706, L251

Cyburt, R. H. , Fields, B. D. and Olive, K. A. 2008, J. Cosmology Astropart. Phys., 0811,012

Cyburt, R. H., \& Davids, B. 2008, Phys. Rev. C, 78,064614

Cyburt, R. H., \& Pospelov, M. 2009, arXiv:0906.4373

Descouvemont, P. 1999, Nucl. Phys. A, 646, 261

Descouvemont, P., Adahchour, A., Angulo, C., Coc, A., \& Vangioni-Flam, E. 2004, Atomic Data and Nuclear Data Tables, 88, 203 astro-ph/0407101.

Efros, V. D., Balogh, W., Herndl, H., Hofinger, R., \& Oberhummer, H. 1996, Zeitschrift fur Physik A Hadrons and Nuclei, 355, 101

Ekström, S., Meynet, G., Chiappini, C., Hirschi, R., \& Maeder, A. 2008, A\&A, 489, 685

Ekström, S., Coc, A., Descouvemont, P., Meynet, G., Olive, K., Uzan, J.-Ph. \& Vangioni, E. 2010, A\&A, 514, A62

Fowler, W. A., \& Hoyle, F. 1964, ApJS, 9, 201

Frebel, A. \& Norris, J.E. 2011, arXiv1102.1748
Fukugita, M., \& Kajino, T. 1990, Phys. Rev. D, 42,4251

Goriely, S., Hilaire, S., Koning, A.J. 2008, A\&A, 487, 767

Gu, X., Boyd, R. N., Farrell, M. M., Kalen, J. D., Mitchell, C. A., et al. 1995, Phys. Lett. B, 343, 31

Guzhovskij, B. J., et al. 1985, Izv. Rossiiskoi Akademii Nauk, Ser. Fiz., 49, 917

Hammache, F., et al. 2010, Phys. Rev. C, 82, 065803

Hashimoto, T., Ishiyama, H., Watanabe, Y. X., Hirayama, Y., Imai, N., Miyatake, H., Jeong, S. C., Tanaka, M.-H., Yoshikawa, N., Nomura, T., Mitsuoka, S., Nishio, K., Sato, T. K., Osa, A., Ichikawa, S., Matsuda, M., Ikezoe, H., Das, S. K., Mizoi, Y., Fukuda, T., Sato, A., Shimoda, T., Otsuki, K., Kajino, T. 2009, Physics Letters B, 674, 276

Heil, M., Käppeler, F., Wiescher, M., \& Mengoni, A. 1998, ApJ, 507, 997

Hernanz, M., Jose, J., Coc, A., \& Isern, J. 1996, ApJ, 465, L27

Hofstee, M. A., Pallone, A. K., Cecil, F. E., McNeil, J. A., \& Galovich, C. S. 2001, Nucl. Phys. A, 688, 527

Igashira, M., Nagai, Y., Masuda, K., Ohsaki, T., \& Kitazawa, H. 1995, ApJ, 441, L89

Iliadis, C. 2007, Nuclear Physics of Stars, by Christian Iliadis. ISBN 978-3-527-406029. Published by Wiley-VCH Verlag, Wenheim, Germany, 2007.,

Iliadis, C., Longland, R., Champagne, A.E., Newton, J.R., Ugalde, C., Coc, A. \& Fitzgerald, R. 2010, Nucl. Phys. A, 841, 31

Iocco, F., Mangano, G., Miele, G., Pisanti, O., \& Serpico, P. D. 2007, Phys. Rev. D, 75, 7304

Iocco, F., Mangano, G., Miele, G., Pisanti, O., \& Serpico, P. D. 2009, Phys. Rep., 472, 1

Ishiyama, H., Hashimoto, T., Ishikawa, T., Watanabe, Y. X., Das, S. K., et al. 2006, Phys. Lett. B, 640, 82 
Izotov, Y. I., \& Thuan, T. X. 2010, ApJ, 710, L67

Kajino, T., Mathews, G. J., Fuller, G. M. 1990, ApJ, 364, 7

Kajino, T. and Boyd, R.N. 1990, ApJ, 359, 267

Kajino, T. 1995, Nucl. Phys. A, 588, 339

Kavanagh, R. W., \& Barnes, C. A. 1958, Phys. Rev., 112, 503

Kawano, L. H., Fowler, W. A., Kavanagh, R. W., \& Malaney, R. A. 1991, ApJ, 372, 1 and 1991, ApJ, 382, 358

Koehler, P. E., \& Graff, S. M. 1991, Phys. Rev. C, 44, 2788

Komatsu, E., et al. 2011, ApJS, 192, 18

Koning, A.J., Hilaire, S. Duijvestijn M. 2008 in Nucl. Data for Science and Technology, (EDP Sciences, eds Bersillon et al.) p 211 (see also http://www.talys.eu)

Lee, H. Y., Greene, J. P., Jiang, C. L., Pardo, R. C., Rehm, K. E., Schiffer, J. P., Wuosmaa, A. H., Goodman, N. J., Lighthall, J. C., Marley, S. T., Otsuki, K., Patel, N., Beard, M., Notani, M., Tang, X. D., 2010, Phys. Rev. C, 81, 015802

The ALEPH Collaboration and The DELPHI Collaboration and the L3 Collaboration and The OPAL collaboration and The SLD Collaboration and the LEP Electroweak Working Group and the SLD Electroweak and Heavy Flavour Groups, 2006, Phys. Rep., 427, 257

Longland, R., Iliadis, C., Champagne, A. E., Newton, J. R., Ugalde, C., Coc, A., \& Fitzgerald, R. 2010, Nuclear Physics A, 841, 1

Malaney, R.A., \& Fowler, W.A. 1989 ApJ, 345, L5

Mizoi, Y., et al. 2000, Phys. Rev. C, 62, 065801

Nagai, Y., Kobayashi, T., Shima, T., Kikuchi, T., Takaoka, K., Igashira, M., Golak, J., Skibiński, R., Wita?a, H., Nogga, A., Glöckle, W. \& Kamada, H. 2006 Phys. Rev. C, 74, 025804

Nollett, K. M., \& Burles, S. 2000, Phys. Rev. D, 61,123505
Paradellis, T., Kossionides, S., Doukellis, G., Aslanoglou, X., Assimakopoulos, P., et al. 1990, Z. Phys. A, 337211

Parpottas, Y., Ahmed, M. W., Blackston, M. A., France III, R. H., Perdue, B. A., et al. 2006, Phys. Rev. C, 74, 015804

Pettini, M., Zych, B.J., Murphy, M., Lewis, A. and Steidel, C.C. 2008, MNRAS391, 1499

Primas, F. 2010, Proceedings IAU Symposium No. 268, Light Elements in the Universe 9-13 November, Geneva, Switzerland, Eds. Corinne Charbonnel, Monica Tosi, Francesca Primas \& Christina Chiappini Cambridge University Press, p. 221

Rauscher, T., Applegate, J. H., Cowan, J. J., Thielemann, F.-K., \& Wiescher, M. 1994, ApJ, 429, 499

Rollinde, E. , Maurin, D., Vangioni, E., Olive, K., Inoue, S. 2008, ApJ, 673, 676

Rollinde, E. , Vangioni, E., Olive, K., 2006, ApJ, 651,658

Ryan, S. G. , Beers, T. C. , Olive, K. A. , Fields, B. D. and Norris, J. E. ApJ, 2000, 530 L57

Sabourov, A., Ahmed, M. W., Blackston, M. A., Crowell, A. S., Howell, C. R., Perdue, B. A., Sabourov, K., Tonchev, A., Weller, H. R., Prior, R. M., \& Spraker, M. C. 2006, Phys. Rev. C, 73, 015801

Sbordone, L., et al. 2010, A\&A, 522, 26

Schmid, G. J., Chasteler, R. M., Weller, H. R., \& Tilley, D. R. 2006, Phys. Rev. C, 48, 441

Serpico, P. D., Esposito, S., Iocco, F., Mangano, G., Miele, G., \& Pisanti, O. 2004, J. Cosmology Astropart. Phys., 12, 10

Siemssen, R. H., Cosack, M., \& Felst, R. 1965, Nucl. Phys. A,, 69, 209

Spergel, D. N., et al. 2007, ApJS, 170, 377

Spite, F. and Spite, M. 1982, A\&A, 115, 357 
Spite, F. and Spite, M. 2010, Proceedings IAU Symposium No. 268, Light Elements in the Universe 9-13 November, Geneva, Switzerland, Eds. Corinne Charbonnel, Monica Tosi, Francesca Primas \& Christina Chiappini Cambridge University Press, p. 201

Steffen, M., Cayrel, R., Bonifacio, P., Ludwig, H.G., \& Caffau, E. 2010, IAU Symposium, 268, 215

Tang, X., Azhari, A., Gagliardi, C. A., Mukhamedzhanov, A. M., Pirlepesov, F., Trache, L., Tribble, R. E., Burjan, V., Kroha, V., Carstoiu, F. 2003, Phys. Rev. C, 67, 015804

Thomas, D., Schramm, D. N., Olive, K. A., \& Fields, B. D. 1993, ApJ, 406, 569

Thomas, D., Schramm, D. N., Olive, K. A., Mathews, G. J., Meyer, B. S., \& Fields, B. D. 1994, ApJ, 430, 291

Vangioni-Flam E. et al 2003, ApJ, 585, 611

Vangioni-Flam, E.; Cassé, M.; Audouze, J. 2000, Phys. Rep., 333, 365

Wagoner, R. V., Fowler, W. A., \& Hoyle, F. 1967, ApJ, 148, 3

Wagoner, R. V. 1969, ApJS, 18, 247

Wang, T. R., Vogelaar, R. B., \& Kavanagh, R. W. 1991, Phys. Rev. C, 43, 883 and 1991, Phys. Rev. C, 44, 1226

Wiescher, M., Görres, J., Graff, S., Buchmann, L., \& Thielemann, F.-K. 1989, ApJ, 343, 352

Wiescher, M., Harms, V., Görres, J., Thielemann, F.-K., \& Rybarcyk, L. J. 1987, ApJ, 316, 162

Xu, Y., Takahashi, K., Goriely, S., Arnould, A. 2010, in Proc. Int. Conf. on Frontiers in Nuclear Structure, Astrophysics and Reactions (eds. Demetriou et al., APS Series), in press

Xu, Y., Takahashi, K., Goriely, S., Utsunomiya, H., Arnould, M., Aikawa, M., Arai K., Katsuma, M., Ohta, M. 2011, Nucl. Phys. A, in preparation

Yamamoto, Y., Kajino, T., \& Kubo, K.-I. 1993, Phys. Rev. C, 47, 846
Yan, J. S., Cecil, F. E., McNeil, J. A., Hofstee, M. A., \& Kunz, P. D. 1997, Phys. Rev. C, 55, 1890

This 2-column preprint was prepared with the AAS IATEX macros v5.2. 